\title{
Die Rolle von kooperativem Lernen und Dramapädagogik in Bezug auf das fremdsprachliche Handeln: Aktionsforschung zum DaF-Theaterprojekt Entre Bastidores mit den Physikern an der Universidad de Salamanca
}

\author{
Theresa Birnbaum
}

\begin{abstract}
Zusammenfassung
Inwieweit kann die Verbindung von kooperativem Lernen (KL) in Theaterprojekten mit der Methode der Dramapädagogik (DP) Wege zum fremdsprachlichen Handeln eröffnen? Am Beispiel des Deutsch-als-Fremdsprache (DaF)-Theaterprojektes Entre bastidores mit den Physikern an der Universidad de Salamanca stelle ich hier eine Aktionsforschung im Bereich der Projektarbeit und des dramapädagogischen Fremdsprachenunterrichts vor. Der ethnografischen Fallstudie lag der Anspruch zugrunde, den Einsatz von Theaterprojekten im DaF-Kontext auf empirischer Ebene zu fundieren, um daraus Handlungsschritte für zukünftige Projekte dieser Art abzuleiten. Ziel der Arbeit war es, die didaktische Gestaltung des Projektes mit Hilfe eines Fragebogens mit offenen Fragen aus der Perspektive der Teilnehmenden (TN) sowie einer Leiterin und einer Beobachterin zu reflektieren. Es ging darum, darzustellen, wie sprachliches Handeln in der Fremdsprache erfolgte und welche Rolle dabei die methodischen Ansätze des KL und der DP spielten. Es wurde dabei von der These ausgegangen, dass sich die Öffnung des Projektes über den institutionellen Rahmen hinaus in einen Lebenskontext sowie die Funktionalisierung der Sprache innerhalb einer projektorientierten und dramapädagogischen Gruppenarbeit positiv auf die Motivation und damit auf den Sprachlern- und Handlungsprozess auswirkten. Das Ergebnis der Studie bildet ein Thesenkatalog mit 17 Hypothesen zum kooperativen und dramapädagogischen Lernen innerhalb des hier vorgestellten Theaterprojektes.
\end{abstract}

\section{Einführung - Das DaF-Theaterprojekt im Sinne eines handlungsorientierten Fremdsprachenlernens}

Gemäß dem Gemeinsamen europäischen Referenzrahmen (GeR) ist das oberste Lernziel des Fremdsprachenunterrichts die Befähigung zum kommunikativen 
Handeln in der Fremdsprache (vgl. Trim et al. 2009: 21). Wenn das Ziel des Fremdsprachenunterrichts die Befähigung der Lernenden zum selbstbestimmten Handeln und zur verstehenden und aktiven Teilhabe an gesellschaftlichen Diskursen in der Fremdsprache ist, dann muss Fremdsprachenunterricht diesen Zielen didaktisch und methodisch begegnen können. Das Klassenzimmer sollte demnach ein Ort sozialer Interaktion sein, an dem die Lernenden miteinander und auch mit dem Lehrer dynamisch interagieren und die Lernumgebung gemeinsam als Lebens- und Erfahrungsraum gestalten.

Zwei methodisch/didaktische Ansätze, die den Anforderungen an einen kommunikativen, handlungsorientierten, ganzheitlich-lebensnahen und lernerzentrierten Fremdsprachenunterricht entsprechen, sind die Konzepte des kooperativen Lernens (KL) und der Dramapädagogik (DP). Eine Möglichkeit, diese beiden Lernformen miteinander zu verknüpfen, sind fremdsprachliche Theaterprojekte, wobei wir die Projektmethode als „Hochform handelnden Lernens" (vgl. Gudjons 2008: 115) verstehen. Ein solches Theaterprojekt initiierte und leitete ich gemeinsam mit meiner Kommilitonin Jana Glaser im Rahmen meines DaF-Masterstudiums im Wintersemester 2010/11 an der Universidad de Salamanca (USAL). Mit diesem Projekt wollten wir Deutschlernenden die Möglichkeit geben, ihre Sprachkenntnisse außerhalb des normalen Unterrichts anzuwenden, gleichzeitig Lust am Theaterspielen wecken und einen internationalen Austausch fördern. Auf diese Initiative hin entstand die internationale Theatergruppe Teatro alemán im Klassenzimmer aus elf Deutschlernenden verschiedener Herkunftsländer und Sprachniveaus sowie aus vier deutschen Studierenden. Das Ergebnis des Projekts war die Inszenierung und anschließende öffentliche Aufführung einer Adaption des Stückes Die Physiker von Friedrich Dürrenmatt, das unter dem Titel Entre bastidores mit den Physikern präsentiert wurde.

Außerdem machte ich dieses Theaterprojekt zum Forschungsgegenstand für eine ethnografische Fallstudie. Ziel der Studie war die Reflexion der didaktischen Gestaltung des DaF-Theaterprojektes. Mit Hilfe eines Fragebogens mit offenen Fragen aus der Perspektive der Teilnehmenden (TN) sowie einer Leiterin und einer Beobachterin sollten Hypothesen über die Wirkungsweisen der Methoden des KL und der DP generiert werden, um darauf aufbauend didaktische Handlungsschritte für zukünftige Projekte dieser Art zu formulieren.

Im Folgenden sollen zunächst die Konstrukte des KL und der DP auf der Grundlage eines kommunikativen und auf sprachliches Handeln ausgerichteten Fremdsprachenunterrichts erläutert werden. Anschließend wird erklärt, warum Lernende im Fremdsprachenunterricht sprachlich handeln sollen und wie diese Ansätze ein solches Lernen begünstigen können. Die Schnittstelle der beiden Lehr-Konzepte wird in der Kombination einer kooperativen Makromethode, dem Projekt, und der Inszenierung eines Theaterstückes auf der Grundlage dramapädagogischer Inszenierungstechniken gefunden. Auf dieser Basis erarbeitete ich ein 10-Punkte-Kriterienkatalog zum KL innerhalb dramapädagogischer Projekte, wodurch die auf das hier vorgestellte Projekt bezogenen didaktischen Handlungsschritte kategorisiert werden konnten und 
somit die Grundlage für das Aktionsforschungsdesign bildeten. Während des Projekt- und Forschungsverlaufs entwickelte ich induktiv Vorab-Hypothesen, die ich dann im Hinblick auf die Fragestellung kritisch überprüfte, erweiterte und modifizierte. Das Ergebnis ist ein Thesenkatalog mit 17 Hypothesen zum kooperativen und dramapädagogischen Lernen innerhalb des hier vorgestellten Theaterprojektes.

\section{Kooperatives Lernen und Dramapädagogik}

Lernen wird innerhalb des Paradigmas des sozialen Konstruktivismus (vgl. Wolff 2002: 19ff) als ein aktiver, konstruktiver Prozess verstanden, bei dem Lernende ihr bereits vorhandenes Wissen mit neugewonnenen Informationen verbinden und auf diese Weise neue Sinnzusammenhänge herstellen. Der Lernende steht dabei mit seinen Vorerfahrungen und Interessen im Zentrum. Der Unterricht sollte sich daher auf Themen beziehen, die die Lebenswelt der Lernenden betreffen, und in eine authentische Lernumgebung eingebettet sein sowie eine kritische Reflexion der Wirklichkeit gewährleisten. Als allgemeines Lernziel eines zeitgemäßen Unterrichts nennt Wolff (vgl. 2002: 22) dabei die Herausbildung von Lernerautonomie. Damit ist die Befähigung der Lerner zum selbstständigen Lernen, zur Bestimmung von Lernzielen und Inhalten, zur Entwicklung und Auswahl entsprechender Lerntechniken und -strategien sowie zur Reflexion von Lernprozessen gemeint. Weiterhin sollte das kollektive Lernen und die Interaktion in Gruppen gefördert werden, um eine Weiterentwicklung und einen Perspektivenwechsel zu garantieren. Der Unterricht sollte Situationen schaffen, in denen Lernende „Sprech-Handeln“ (Mairose-Parovsky 2000: 60ff) können und das in einem ganzheitlichen Sinne. Schließlich sollten affektive und motivationale Faktoren berücksichtigt werden, indem Gefühle und persönliche Identifikation einbezogen werden.

An dieser Stelle setzen handlungsorientierte Konzepte an. Wenn Lernende und Lehrende den Unterricht nicht als eine von der Außenwelt abgeschottete Institution betrachten, sondern als Teil ihrer Lebenswelt, dann wird Fremdsprachenlernen zum Bestandteil eines Sozialisationsprozesses, bei dem nicht nur die Strukturen einer fremden Sprache, sondern gleichzeitig kulturelle und soziale Faktoren vermittelt werden und eine persönliche Weiterentwicklung stattfindet. Gehen wir davon aus, dass Lernende in ihrer realen Lebenswelt ihre Muttersprache im Sinne einer echten kommunikativen Funktion nutzen, also sie zur Erreichung eines persönlichen oder umweltgerichteten Handlungszieles einsetzen, dann sollte Fremdsprachenunterricht die gleichen Zwecke verfolgen. Das bedeutet, er muss sich diesen lebensnahen Kommunikations- und Lernprozessen öffnen, indem er didaktische Situationen schafft, die entweder unmittelbar in die Realität eingreifen oder als lebensecht akzeptiert werden können und in denen Lernende für sie reale und ihre eigene Lebens- und Erfahrungswelt betreffende Kommunikationsziele verfolgen. Gudjons beschreibt diesen Prozess mit dem Begriff des „kommunikativen Handelns“ (2008: 42), das er als zentrales Element schulischen Erfahrungslernens bezeichnet. 


\subsection{Kooperatives Lernen}

Da Lernen ein konstruktiver und sozialer Prozess ist, der auf Interaktions- und Kommunikationshandlungen zwischen den Lernenden beruht, ist der ideale Lernkontext ein kooperativer, also eine Umgebung, die gemeinschaftliches Lernen fördert. Denn KL bietet „eine Interaktionsform, bei der die beteiligten Personen gemeinsam und in wechselseitigem Austausch Kenntnisse und Fertigkeiten erwerben“ (Konrad \& Traub 2005: 5). Die Literatur beschreibt KL in den meisten Fällen als ein Lernen in Kleingruppen innerhalb eines institutionellen Unterrichts: „Cooperative learning is the instructional use of small groups so that students work together to maximize their own and each other's learning" (Johnson \& Johnson 1994: 107).

Dieser Gedanke soll allerdings im Hinblick auf das hier vorgestellte Projekt erweitert werden. Es wird davon ausgegangen, dass KL sowohl in Kleingruppen als auch in Großgruppen und innerhalb sowie auch außerhalb eines institutionellen Rahmens stattfinden kann. Bonnet \& Küppers (2011: 38) beschreiben das Lernen in Großgruppen als ,kooperative Makromethode', zu der auch die Projektmethode zählt:

Kooperative Makromethoden erfordern die Auseinandersetzung mit lebensweltlich komplexen (Storyline) oder auch komplexen und für die Lernenden nicht alltäglichen Problemen (z.B. Simulation) und die Bearbeitung zwischenmenschlicher Probleme.

Auf den Fremdsprachenunterricht bezogen können kooperative Makromethoden helfen, fremdsprachliches Handeln authentischer und plausibler zu gestalten, indem die Schüler fiktionale Rollen in realitätsbezogenen Kontexten annehmen.

\subsection{Dramapädagogik als ganzheitliche Methode}

Eine Möglichkeit, kooperativ in lebensnahen Kontexten zu lernen und dabei in der Fremdsprache zu handeln, bietet die Verbindung von Fremdsprachenunterricht und Theater. Bonnet \& Küppers beschreiben die DP als eine „Methode, die sich ausgewählter Elemente aus dem Bereich des Theaters und Schauspiels bedient, um sie für Bildungszwecke nutzbar zu machen" (2011: 41). Als pädagogisches Unterrichtsprinzip meint dies zunächst den „kreative[n] Umgang mit dramatischen Texten“ und eine „handlungsorientierte Aneignung von Dramentexten“ (Koch \& Streisand 2003: 81). Schewe (1993: 4) sieht die Verbindung zwischen DP und Fremdsprachenunterricht in der Förderung kommunikativer Handlungskompetenzen durch eine stärkere künstlerische Orientierung:

Mit Hilfe von Methoden, die sich aus der dramatischen Kunstform ableiten lassen, werden im Unterricht fiktive Kontexte geschaffen, in denen Lehrende und Lernende sprachlich und nichtsprachlich in intensiver Weise handeln - die fremde Sprache wird inszeniert. 
Bei der DP geht es darum, innerhalb des „realen Schulkontextes“ eine fiktive Wirklichkeit, eine sogenannte „Als-ob“-Situation zu schaffen, in der Lernende sprachlich spontan handeln und sich ausprobieren können. Ziel ist es, durch ein Sich-Hineinversetzen in andere Rollen und Situationen, durch ein „So-tun-als-ob“, andere Perspektiven zu übernehmen und Sachverhalte oder Konflikte von innen heraus zu untersuchen. Hierbei spielen Empathie und Reflexion eine zentrale Rolle. Es geht dabei nicht in erster Linie um das Entwickeln von schauspielerischen Fähigkeiten, sondern eher um den Prozess des sozialen und kooperativen Lernens sowie die Entwicklung von Problemlösestrategien, die Fähigkeit zur Reflexion und zum kritischen Denken und die Förderung kommunikativer Kompetenzen (vgl. Bonnet \& Küppers 2011). Dramapädagogischer Fremdsprachenunterricht geht im Sinne reformpädagogischer Ideen von einem ganzheitlichen Lernen aus, bei dem kognitive, emotionale und motorische Fähigkeiten verbunden werden und das Individuum ins Zentrum rückt. Das Lernen wird als „einheitlicher, zusammenhängender Erlebnisprozess, an dem das lernende Individuum aktiv teilnimmt" (Schlemminger 2000: 16) verstanden. Für Schewe bedeutet Ganzheitlichkeit die „Verbindung geistlicher Erkenntnisse mit sinnlichem Genuss" (1993: 75). Anknüpfend an die Maxime Pestalozzis spricht er von einem Lernen mit Kopf, Herz und Hand und - wie er betont - auch mit dem Fuß (vgl. 1993: 7). Er erklärt wie Sprechhandeln im dramapädagogischen Fremdsprachenunterricht funktioniert:

\begin{abstract}
Lehrende und Lernende werden im dramapädagogischen Fremdsprachenunterricht zu Konstrukteuren von Handlungssituationen. Mit Hilfe entsprechender Inszenierungstechniken werden fiktive Kontexte geschaffen, die so konstruiert sind, dass die Beteiligten nicht anders können als (körperlich) zu handeln. Und aus der Handlung heraus formiert sich die (fremde) Sprache.(Schewe 1993: 409)
\end{abstract}

\title{
2.3 Die Schnittstelle von kooperativem Lernen und Dramapädagogik nach Kriterien der Handlungsorientierung
}

Ausgehend von diesen Vorüberlegungen wird im Folgenden ein Kriterienkatalog vorgestellt, der zehn wesentliche Lernziele kooperativen und dramapädagogischen Lernens vereint:

Lernerorientierung: Kooperative Makromethoden beschäftigen sich mit komplexen, lebensnahen Themen und beziehen dabei die Vorerfahrungen und Interessen der Lernenden mit ein. So werden beispielsweise bei Storyline oder Simulation authentische Situationen nachgespielt. Dramapädagogischer Unterricht setzt sich in fiktiven Kontexten mit für die Lernenden relevanten Themen und Fragestellungen auseinander.

Lernerautonomie: Kooperative Makromethoden fördern die Selbstbestimmung der Lernenden, indem diese für ihr Lernen und das der anderen Verantwortung übernehmen müssen und diesen Prozess aktiv steuern und reflektieren. Dazu gehört auch, sprachliche Handlungsprodukte zu evaluieren 
und selbstständig bzw. mit Hilfe der Mitlernenden zu korrigieren. Der Lehrende ist dabei je nach Interaktionsform mal vermittelnder Moderator und Hilfesteller, mal als Akteur oder Impulsgeber in das Rollenspiel involviert.

Kooperatives und soziales Lernen: Dramapädagogische Übungsformen finden in der kommunikativen Interaktion mit anderen statt (face-to-faceInteraktion) und fördern die positive Interpendenz (wechselseitige Abhängigkeit, vgl. Borsch 2010:19) durch gemeinsame Lernziele, interaktive Gruppenaktivitäten (z.B. gemeinsames Arbeiten an einem Standbild) und durch den Konsens, sich auf die fiktive Wirklichkeit einzulassen. Eine entscheidende Rolle spielt dabei die Fähigkeit, eine fiktive Rolle einzunehmen und so andere Perspektiven zu übernehmen und Handlungsschritte zu erproben. DP fördert das Voneinander-Lernen und die gegenseitige Hilfestellung, insbesondere in heterogenen Lernergruppen. Dies erfordert den Einsatz sozialer Kompetenzen wie Feedbackfähigkeit (ein kommunikativer Prozess, bei dem sich die Akteure eines Gruppenprozesses darüber unterhalten, was sie an anderen Personen wahrgenommen haben und gleichzeitig selbst mit Beobachtungen über ihr eigenes Verhalten konfrontiert werden - vgl. Koch\&Streisand 2003:98) und die Übernahme von individueller Verantwortlichkeit sowie die Bereitschaft, sich ernsthaft mit dem Lerngegenstand auseinanderzusetzen.

Kommunikation und sprachliches Handeln: DP schafft durch fiktionale „Als-ob-Situationen“ kommunikative Handlungsräume, in denen sich die Lerner sprech-handelnd ausprobieren können, ohne reale Konsequenzen für ihr sprachliches Handeln befürchten zu müssen (vgl. Schewe 1993: 401). Durch die Interaktion im Rollenspiel und das Simulieren von sogenannten „Sprachnotsituationen“, wie sie Tselikas (1999: 41) beschreibt, werden Sprechimpulse geschaffen und echte Redeanlässe simuliert.

Ganzheitlichkeit im dramapädagogischen Prozess: Bei dramapädagogischen Übungen findet Lernen mit allen Sinnen statt. Wie im echten Leben vollzieht sich Sprachenlernen unter Einbeziehung von Körperlichkeit, Bewegung und Sinnlichkeit sowie unter Einsatz der Stimme. Das Klassenzimmer wird dabei zum Erfahrungsraum, der im Sinne der fiktionalen Handlung umfunktioniert und kreativ genutzt wird.

Reflexion und Evaluation: Die Reflexion des Gruppenprozesses ist ein Teil der DP, um „zum Kern des Lerngegenstandes vorzustoßen und dadurch Bildungsprozesse anzuregen“ (Bonnet \& Küppers 2011: 47). Aus der Reflexion sollen Handlungsschlüsse gezogen werden, die in der nächsten Aktion umgesetzt werden. Dabei geht es auch im Sinne der Lernerautonomie um die Fähigkeit, eigene Lernprozesse zu reflektieren, und um die Kompetenz, konstruktives Feedback zu geben und zu erhalten.

Emotionale und motivationale Faktoren: Gefühle und persönliche Identifikation spielen beim dramapädagogischen und kooperativen Lernen eine entscheidende Rolle. Zum einen sind sie unmittelbarer Bestandteil einer jeden dramatischen Situation und können so zur Reflexion von lernerrelevanten Themen genutzt werden, ohne zu persönlich zu werden. Zum anderen dienen sie als Sprechanlass, um auf eigene Bedürfnisse einzugehen. Ein weiterer 
wichtiger Faktor ist, dass das Lernen in einer Gruppe und das Sich-Ausprobieren in spielerischen Kontexten die Motivation und den Spaß am Lernen fördern und Stress sowie Sprechhemmungen abbauen.

Performative Kompetenz: Performative Kompetenz ist die Fähigkeit, sich kritisch mit der „Inszeniertheit“ (vgl. Hallet 2010) von Wirklichkeit auseinanderzusetzen und sie im Spiel selbst zu ergründen. Partizipation wird im Sinne der DP sowohl im Spielen als auch im Zuschauen begründet, da das reflektierende Beobachten gleichzeitig eine Teilnahme an den Lernprozessen ist.

Gesellschaftliche Teilhabe und Demokratiegedanke: Kooperative Makromethoden, besonders die Spezialform Projekt, bieten die Möglichkeit, in die gesellschaftliche Wirklichkeit verändernd einzugreifen und die Grenzen des Klassenzimmers zu öffnen. Zunächst geht es darum, gesellschaftliche Prozesse zu verstehen und an sozio-kulturellen Diskursen teilzuhaben. Die Lernenden sollen aber auch die Möglichkeit bekommen, die Entscheidungen und Organisationen innerhalb des Klassenzimmers im Sinne eines Demokratiegedankens mitzutragen. Hierzu gehören z.B. das gemeinsame Aufstellen von Regeln, das gemeinsame Finden einer Projektidee etc.

Produktorientierung: Dramapädagogische Makromethoden und KL sollten Handlungsergebnisse hervorbringen, die den Lernprozess widerspiegeln, die für die Lernenden von Bedeutung sind und die sie einander oder vor einem Publikum präsentieren können. Damit werden die Produkte kommunizierbar, also der Kenntnisnahme und Kritik anderer zugänglich gemacht (vgl. Gudjons 2008: 86). Produkte können verschiedene Formen haben (aktional, z.B. Podiumsdiskussion; performativ, z.B. eine Theateraufführung; dokumentarisch, z.B. eine Broschüre; ausstellerisch, z.B. Stellwände gestalten etc.). Sie dienen dazu, eine Problemlösung an der Wirklichkeit zu überprüfen sowie Wissen fassbar zu machen, und sie haben eine motivationale Funktion (vgl. ebd. 88f).

\section{Das Theaterprojekt Entre bastidores mit den Physikern - Ziele und didaktische Handlungsschritte}

Das DaF-TheaterprojektEntre bastidores mit den Physikern baute didaktisch und methodisch auf diesen zehn Kriterien auf. Das übergeordnete Projektziel war es, gemeinsam mit den fünfzehn internationalen Teilnehmenden (TN) eine eigene Version des Stückes Die Physiker von Friedrich Dürrenmatt zu inszenieren und diese am Ende des Semesters vor einem Publikum zur Aufführung zu bringen. Der Projektzeitraum erstreckte sich von Anfang Oktober bis Mitte Dezember 2010 und enthielt insgesamt 26 Theaterprobenstunden. Außerhalb der Proben traf sich die Gruppe auch zu einzelnen Aktivitäten wie Stammtischen, Geburtstagsfeiern, zum Videoabend und zu Einzelproben im Café, in einer Kneipe oder auch im Wohnzimmer eines Mitglieds. Die Aufführung fand auf einer professionellen Theaterbühne im Palacio Fonseca in der Stadt Salamanca statt. 
Der Erfolg eines handlungs- und produktorientierten Projektes basiert darauf, dass die Lehrziele der Lehrenden mit den Handlungszielen der Lernenden weitestgehend übereinstimmen sollten (vgl. Gudjons 2008: 149). Es ging uns als Projektleiterinnen also vor allem darum, die von uns gesteckten Ziele den Erwartungen und Interessen der Lernenden anzupassen. Um herauszufinden, welche Bedürfnisse die TN hatten, führten wir daher regelmäßig Evaluationsrunden durch und baten auch um schriftliches Feedback in der E-Mail-Kommunikation. Weiterhin besprachen wir uns regelmäßig und reflektierten den Projektprozess.

Im Folgenden werden die von uns arrangierten didaktischen Handlungsschritte in den in Punkt 2.3 vorgestellten Kriterienkatalog für dramapädagogischkooperatives und handlungsorientiertes Fremdsprachenlernen verankert. Dies geschieht zunächst in Form einer deskriptiven Retrospektive aus der Eigenperspektive als Projektleiterin und begründet sich auf der Projektdokumentation. Angemerkt sei, dass sich die Zuordnung der didaktischen Handlungsschritte in die Kriterien an manchen Stellen problematisch gestaltet, da sich viele Faktoren gegenseitig beeinflussen.

Lernerorientierung: Im Zentrum des Projektes standen die TN. Sie gestalteten das Theaterprojekt mit ihren Ideen selbst. Als Projektleiterinnen wollten wir erreichen, dass sich alle Lernenden mit dem Projekt identifizieren können und ein Interesse daran entwickeln - zum einen auf der inhaltlich-künstlerischen Ebene, zum anderen auf der gesellschaftlich-sozialen Ebene. Außerdem war es uns wichtig, dass sich alle gemäß ihren Deutschkenntnissen und Theatererfahrungen in den Prozess einbringen konnten. Unsere Aufgabe war es deshalb, die Stärken und Schwächen jedes Einzelnen zu erkennen, Ängste und Unsicherheiten in Reflexionsrunden und persönlichen Gesprächen zu hinterfragen und so jeden TN auch individuell zu erreichen. Dazu war es nötig, ein Vertrauen innerhalb der Gruppe aufzubauen, das es jedem Gruppenmitglied erlaubt, er oder sie selbst zu sein, sich einzubringen, Probleme anzusprechen und nachzufragen. In Bezug auf das Deutschniveau der TN hatten wir es mit unterschiedlichen Niveaustufen und Lernerbiografien zu tun. Hinzu kam die Konstellation der Zusammenarbeit zwischen deutschen Muttersprachlern (MS) und Nichtmuttersprachlern (NMS). Eine der größten Herausforderungen war es daher, gemäß einer Binnendifferenzierung alle TN mit ihren Stärken und Schwächen in das Projekt einzubeziehen und zu fördern. Keiner sollte sich bei der Arbeit über- oder unterfordert fühlen und alle sollten das Stück verstehen und dem Probenprozess folgen können. Aus diesem Grund näherten wir uns zunächst dem Stück in einer ersten interaktiven Sitzung, in der wir zunächst eine Einführung in den Inhalt und die Rollenkonstellationen gaben und schließlich eine Leseprobe durchführten. Danach besprachen wir in Kleingruppenarbeit Schlüsselsätze aus dem Stück und stellten diese anschließend im Plenum vor. Für die Lektüre der Texte war es wichtig, dass die TN sich mit der Problematik des Stückes auseinandersetzen und auch die Dramentheorie von Dürrenmatt kennenlernten. Dadurch fiel es ihnen leichter, den Text auch metasprachlich zu erschließen. Auf der künstlerischen Ebene der Stückentwicklung nutzten 
wir einen dramaturgischen Kunstgriff, um den TN möglichst viel Raum zur Gestaltung und zur Einbringung eigener Ideen zu geben. Wir schlugen der Gruppe vor, eine zweite Spielebene einzuführen, auf der sich - ähnlich wie in der Lebenswelt der TN - eine studentische Theatergruppe trifft, um Die Physiker von Dürrenmatt einzustudieren. Der Gedanke war es, ein Stück im Stück aufzuführen, nämlich eine Probe des Stückes Die Physiker zu zeigen. Hierbei sollten auch die Höhen und Tiefen sowie zwischenmenschliche Probleme, die so ein Projekt mit sich bringen kann, thematisiert werden. Gleichzeitig erlaubte uns dieser Kunstgriff, den anspruchsvollen Text von Dürrenmatt auf eine lernerfreundliche Fassung zu kürzen und ihn innerhalb des Probencharakters des Stückes als Sprechhilfe mit auf die Bühne nehmen zu lassen. Diese Idee stellte gleichzeitig einen Bezug zur Realität dar, da sich die Gruppe ebenfalls in einer solchen Situation befand und die TN auf ihre persönlichen Erfahrungen während des Probenprozesses zurückgreifen und diese im Spiel reflektieren konnten. Außerdem bot sie den TN vor allem die Möglichkeit, diese Ebene selbst zu gestalten, ihre eigenen Rollen darin zu entwickeln und sprachlich zu handeln. Sprachliche Tätigkeit äußerte sich hier auch darin, dass die TN an ihren eigenen Sprachprodukten arbeiteten und diese weiterentwickelten. Beispielsweise überarbeiteten wir gemeinsam Rollentexte, übten die Aussprache oder kürzten den Dürrenmatt-Text. Auf diese Weise fand eine intensive Spracharbeit und metafiktionale Reflexion der Rollentexte statt.

Lernerautonomie: Lernerautonomie meint hier vor allem die Übernahme von Verantwortung für das eigene Handeln und den selbstgesteuerten Sprachund Lernprozess innerhalb des Projektes. Sie geht Hand in Hand mit Lernerorientierung. Zunächst ging es hier darum, dass die Lernenden an der kreativen Gestaltung des Projektes, wie oben beschrieben, aktiv mitarbeiten konnten. Daher gaben wir den TN die Möglichkeit, ihre Rollenbiografie selbst zu schreiben, Kostüme selbst zu gestalten und auch den Inhalt des Stückes voranzutreiben. Dabei boten wir unsere Hilfe an, in Form von Einzelproben zur Texterschließung, Aussprache und Rollenentwicklung sowie Korrekturen und Tipps per E-Mail. Dieses Angebot nahmen viele TN wahr. Ein methodisches Mittel, um Lernerautonomie zu fördern, war eine ständige E-Mail-Korrespondenz zwischen den Projektleiterinnen und den TN. Auf diese Weise konnten wir die Gruppe über Neuigkeiten informieren und waren immer für Fragen und Korrekturen etc. erreichbar. Außerdem versuchten wir, die TN bei fast allen Entscheidungen, die das Projekt betrafen, mit einzubeziehen. Dazu gehörte auch die aktive Beteiligung an der Organisation der Rahmenbedingungen und an der Planung der einzelnen Handlungsschritte (wie Terminabsprachen, Raumplanung).

Kooperatives und soziales Lernen: Das Projekt bot den idealen Kontext zum kooperativen und sozialen Lernen. Das Projektziel konnte nur erreicht werden, da sich alle aktiv in den Prozess einbrachten und auf die Theateraufführung gemeinsam hinarbeiteten (positive Interpendenz). Dadurch, dass sich die Gruppenmitglieder auf den fiktiven Theaterprozess einließen, wurden mehr und mehr Hemmungen in Bezug auf das Spielen und auch das Sprechen 
abgebaut. Dabei ergänzten sich die TN nicht nur im Hinblick auf ihr unterschiedliches Deutschniveau, sondern auch in Bezug auf ihre persönlichen Eigenschaften. So gab es NMS, die zwar sprachlich ein hohes Niveau hatten, denen es aber schwer fiel, laut zu sprechen oder frei auf einer Bühne $\mathrm{zu}$ improvisieren. Durch zahlreiche interaktive Theaterspielübungen, d.h. dramapädagogische Inszenierungsformen und sprachliche Übungsformen (z.B. schauspielerische Improvisationsübungen, phonetische Übungen, Erwärmung von Körper und Stimme, Raumerfahrung usw.) versuchten die Spielleiterinnen daher, zunächst eine Gruppenidentität und -dynamik aufzubauen, die es den TN zunehmend erleichterte, sich einzubringen, aber auch um Hilfe zu bitten. Durch die Zusammenarbeit von Deutschlernenden mit deutschen MS fand außerdem authentisches Sprachenlernen statt, da die Lernenden die ganze Zeit mit der deutschen Sprache in Kontakt waren. Zu authentischen Kommunikationssituationen äußert sich Tschirner (2004:48) folgendermaßen:

Es ist unklar, welche Aspekte kommunikativer Sprachverwendung unter
welchen Umständen auf welche Weise zu erhöhter sprachlicher Kompe-
tenz führen. Deshalb sollte man davon ausgehen, dass nur authentisch
verwendete Sprache, im Lehrer-Schülergespräch, im Austausch mit Mut-
tersprachlern sowie in Radio- und Videosendungen, ein Inputangebot lie-
fert, das reichhaltig genug ist, kommunikative Kompetenz zu erwerben.

Sowohl MS als auch Lerner mit fortgeschrittenen Deutschkenntnissen konnten schwächere Lerner sprachlich unterstützen. ObwohldieProjektsprachemeistens Deutsch war, wurde gerade anfangs auch viel ins Spanische übersetzt bzw. auf Spanisch gesprochen, was wiederum von den deutschen Austauschstudenten als sehr bereichernd reflektiert wurde und die spanischen MS in die Rolle der Lehrenden versetzte.

Kommunikation und sprachliches Handeln: Der zentrale Aspekt beim sprachlichen Handeln ist es, dass die Sprache als Mittel einem Handlungszweck dient, und genau das war bei unserem Projekt der Fall. Nicht das Deutschlernen stand hier im Vordergrund, sondern die Idee, gemeinsam ein deutsches Theaterprojekt zu gestalten, bei dem die Hauptverkehrssprache Deutsch ist und jeder TN gemäß seinem Niveau seine Deutschkenntnisse praktisch und kreativ anwenden und verbessern konnte. Es ging also darum, einen kommunikativen und authentischen Handlungskontext zu schaffen, innerhalb dessen die Sprache in der Anwendung gelernt wird. Zudem bot die von uns geschaffene Metaebene des Stückes die Möglichkeit, sprachliches Handeln in einer fiktiven Rolle zu testen und sich spielerisch auszuprobieren sowie Handlungsabläufe sprachlich zu reflektieren. Viele Theaterspielübungen zielten am Anfang darauf ab, in der Fremdsprache zu improvisieren und Impulse zum freien und kreativen Sprechen zu geben.

Ganzheitlichkeit im dramapädagogischen Prozess: Ein Theaterprojekt ist ein Paradebeispiel ganzheitlichen Lernens. In den Theaterspielübungen nutzten wir den ganzen Körper zum Sprechen. Dabei flossen sowohl non-verbale als auch phonetische Übungen ein. Das Klassenzimmer wurde dabei mit Hilfe unsere Phantasie zum Bühnenraum, den wir immer wieder neu beschrieben und 
entdeckten. Zudem wurden aufgrund der unterschiedlichen Aufgabentypen alle vier Fertigkeitsbereiche angesprochen. Mal mussten die Akteure eine schriftliche Rollenbiografie verfassen, mal mündlich improvisieren, mal zuhören und beobachten oder einen Rollentext kürzen. Im Sinne eines ganzheitlichen Deutschlernens gestalteten sich aber auch die Treffen außerhalb der Proben. Dem Projekt lag eine gemeinsam gestaltete Erfahrungswelt zugrunde, in die die TN emotional und persönlich eingebunden waren. So fand Sprachenlernen beim gemeinsamen Kaffeetrinken, auf Geburtstagsfeiern und beim Schauen des Premierenvideos in einem ganzheitlich-sinnlich-körperlichen Lernkontext statt.

Reflexion und Evaluation: Ein zentraler Punkt der Lernerautonomie und der sprachlichen Teilhabe waren die regelmäßig stattfindenden Reflexionsrunden. Dies geschah teilweise zu Beginn der Proben, wo alle TN noch einmal ihre Vorstellungen, Probleme und Gedanken äußern konnten, manchmal in Form eines ,Abschlussblitzlichtes' und auch in freier Form bei den außerinstitutionellen Treffen. Besonders bei diesen Gelegenheiten konnten die Projektleiterinnen informelles Feedback bekommen und Kritik wahrnehmen, um darauf entsprechend zu reagieren. Auf diese Weise konnten sie feststellen, wo es Probleme mit dem Textlernen gab, wo sich TN gestresst fühlten oder das Gefühl hatten, nicht weiterzukommen. Es ging aber auch darum, den Gesamtprozess zu reflektieren, das eigene Lernen zu evaluieren und den anderen Gruppenmitgliedern Feedback zu geben und dadurch das Gruppengefühl zu stärken.

Emotionale und motivationale Faktoren: Emotionale und motivationale Faktoren spielten bei dem Projekt eine entscheidende Rolle. Es war es wichtig, dass alle Beteiligten Interesse an dem Projekt entwickelten und das Gefühl hatten, daraus einen persönlichen Mehrwert zu ziehen. Die Erwartungen, die an das Projekt geknüpft waren, sahen bei jedem anders aus und wurden in dem hier beschriebenen Forschungsprojekt hinterfragt. Um eine Identifikation mit dem Projekt zu gewährleisten, war es die Aufgabe der Spielleiterinnen, ein positives Gruppengefühl zu schaffen. Innerhalb der Proben dienten hierzu die gruppendynamischen Theaterspielübungen. Die Möglichkeit, sich in spielerischen und ungezwungenen Kontexten, die nicht benotet werden, auszuprobieren, sollte dabei den Spaß an der Lernform Theater fördern und Stress sowie Sprechhemmungen abbauen. Außerhalb der Proben boten die zusätzlichen Aktivitäten und Treffen einen Raum zum Kennenlernen und trugen dazu bei, dass sich jeder als Teil der Gruppe fühlt. Wichtig war außerdem, dass sich alle in den Projektprozess einbringen konnten. Darum fanden alle Ideen Gehör und bekamen Raum zur Diskussion. Weiterhin wurden Einzelaufgaben verteilt (z.B. was die Kostümbeschaffung betraf) und gemeinsam erfanden wir einen Gruppennamen und den Titel des Stückes.

Performative Kompetenz: Dem Bildungsziel, Performative Kompetenz auszubilden, versuchten wir insofern nachzukommen, indem wir sowohl das Stück Die Physiker als auch unsere eigene Inszenierung und die darin vorkommenden fiktiven Ebenen immer wieder metafiktional besprachen und weiterentwickelten. Die Beteiligten sollten ein Verständnis von der Inszenierung von 
Wirklichkeit bekommen, indem sie die theatralen Prozesse reflektierten und die Texte auch metasprachlich erschlossen. Zu diesem Prozess gehörten auch das gemeinsame Schauen und die Auswertung des Premierenvideos sowie eine Abschlussreflexion mit externen Zuhörern und Zuschauern.

Gesellschaftliche Teilhabe und Demokratiegedanke: Ziel des Projektes war es, die TN zu Akteuren und Mitgestaltern eines Stückes sozialer Wirklichkeit zu machen. Dazu wurden auf der Ebene der Projektarbeit Möglichkeiten geschaffen, um alle aktiv in den Prozess einzubeziehen. Die Öffnung des Projektes über den institutionellen Rahmen hinaus und die Chance, das fertige Theaterstückvor einem externen Publikum in einem öffentlichen Theatersaalder Stadt Salamanca zu präsentieren, gab dem Projekt zusätzliche gesellschaftliche Bedeutung. Die TN lieferten mit der Aufführung eines deutschen Theaterstückes einen Beitrag zum kulturellen Leben Salamancas. Sie veränderten damit ein Stück universitäre und gesellschaftliche Wirklichkeit. Sie selbst waren Akteure und konnten das Ergebnis ihrer Arbeit in der Zeitung finden und auf Plakaten in der Stadt sehen. In einer Abschlussreflexion mit anderen Studenten und Dozenten der deutschen Philologie hatte die Theatergruppe außerdem die Möglichkeit, sich dem Feedback und den Fragen der Zuschauer zu stellen und ein eigenes Fazit zu ziehen.

Produktorientierung: Kleine und große Produkte begleiteten den Projektprozess. Das übergeordnete Endprodukt, das Theaterstück, entstand auf der Grundlage vieler Zwischenergebnisse, die von den Akteuren selbst gestaltet wurden, wie beispielsweise Rollenbiografien, Standbilder, Szenen und Dialoge. Die Produkte wurden durch Aufgaben und Übungen von den Projektleiterinnen initiiert und angeregt, dann aber von den Akteuren selbst hergestellt und in der Interaktion weiterentwickelt. Sie stellten dabei eine wichtige Grundlage für die Reflexion des Projektprozesses dar und waren ebenfalls Zweck sprachlichen Handelns.

\section{Das Forschungsprojekt - Ziele und Erkenntnisinteresse}

Das Theaterprojekt Entre bastidores mit den Physikern an der USAL bildete die Grundlage der hier vorgestellten Aktionsforschung. Diese hatte zum Ziel, die Lernprozesse, die innerhalb des Projektes stattfanden, zu untersuchen und darzustellen, wie sprachliches Handeln in der Fremdsprache erfolgte und welche Rolle dabei die methodischen Ansätze des KL und der DP spielten. Es wurde von dem Ansatz ausgegangen, dass das Lernen einer Fremdsprache nicht nur der Zuwachs sprachlicher Wissensstrukturen ist, sondern innerhalb gesellschaftlicher und sozialer Kontexte stattfindet und ein konstruktiver Prozess ist. Dabei erfolgt das Fremdsprachenlernen durch sprachliches Handeln, wobei Projekte in der Fremdsprache, in diesem Fall über die Methode des Theaterspiels, dieses Lernen positiv fördern können. Außerdem wurde die These vertreten, dass sich die Funktionalisierung der Sprache durch die Öffnung des Projektes über den institutionellen Rahmen hinaus in einen Lebenskontext sowie innerhalb einer projektorientierten und 
dramapädagogischen Gruppenarbeit positiv auf die Motivation und damit auf den Sprachlern- und Handlungsprozess auswirkte. Ziel der Forschung war es, Hypothesen auf die Forschungsfrage "Welche Rolle spielten kooperatives Lernen und Dramapädagogik in Bezug auf das fremdsprachliche Handeln innerhalb des Theaterprojektes Entre bastidores mit den Physikern?" zu generieren.

\subsection{Entwicklung der Vorab-Hypothesen}

Um das Forschungsziel zu erreichen, formulierte ich in einem ersten Schritt folgende Fragen im Hinblick auf die methodisch/didaktischen Lernziele, die sich aus der Beobachtung, Reflexion und Dokumentation des Projektverlaufes ergaben:

- Was waren die Erfolgsfaktoren, die das Projekt als Verknüpfung von Fremdsprachenlernen und Theater auszeichneten, und kann man diesen Erfolg beschreiben?

- Was motivierte die TN, sich in dem Projekt zu engagieren?

- Welche Rolle spielten emotionale und motivationale Faktoren innerhalb des Projektes?

- Durch welche Faktoren zeichnete sich die Gruppenarbeit aus?

- Wie interagierten die TN sprachlich untereinander und mit den Projektleiterinnen?

- Welche Rolle spielte das didaktisch/methodische Arrangement der Projektleiterinnen in Bezug auf die Lernprozesse, die während des Projektes abliefen?

- Kann man einen sprachlichen und persönlichen Lernerfolg durch die Projektarbeit beschreiben und begründen?

Diese Fragen bildeten den Ausgangspunkt für die Formulierung von Vorannahmen über den Lernprozess, welche ich während der Durchführung des Projektes im Sinne eines ethnografischen Forschungsansatzes beobachtete. Mithilfe von multipel erhobenem Dokumentationsmaterial aus unterschiedlichen Perspektiven (Videomitschnitte der Proben und Evaluationsrunden, Probennotizen der Projektleiterinnen, Reflexionsgespräche der Projektleiterinnen mit der Beobachterin, Probenprodukte der TN etc.) formulierte ich folgende auf das Projekt bezogene „Ex-ante-Hypothesen“ (nach Meinefeld 2008: 266, hier als Vorab-Hypothesen bezeichnet), auf die sich die Konzeption des offenen Fragebogens stützt:

- Das kooperative Lernen innerhalb des Projektes förderte die sprachliche Interaktion zwischen den TN. Dadurch konnten die Quantität und Qualität fremdsprachlicher Äußerungen erhöht und Sprechhemmungen abgebaut werden. 
- Dramapädagogik eignete sich als ganzheitliche Methode besonders, um fremdsprachliches Handeln zu fördern.

- Das kooperative Lernen innerhalb der Gruppe steigerte die Motivation eines jeden Einzelnen, sich aktiv in das Projekt einzubringen und trug damit zum Erfolg des Projektes und zum persönlichen Lernerfolg bei.

- Die Möglichkeit, innerhalb des Projektprozesses selbst sprachlich zu handeln und Verantwortung zu übernehmen, förderte das autonome Lernen der TN.

- Das Endprodukt, die gemeinsame Theateraufführung vor Publikum, wurde von vielen TN als sehr wichtig für die Motivation und das persönliche Engagement eingestuft.

- Die Motivation jedes Einzelnen und der Zusammenhalt der Gruppe wurden positiv durch die gesellschaftliche Öffnung des Projektes über die Theaterproben hinaus, von einem Lern- in einen Lebenskontext, beeinflusst.

- Die Zusammenarbeit von MS und NMS sowie TN unterschiedlicher Sprachniveaus und Herkunftsländer wurde von den TN als bereichernd und lernfördernd empfunden.

Auf diesen Vorab-Hypothesen aufbauend wurde ein offener Fragebogen entwickelt, mit dem die Probanden das Projekt rückblickend auswerten konnten. Damit leisteten sie einen wichtigen Beitrag zur Generierung von Hypothesen über die Erfolgskriterien des Theaterprojektes und zur Formulierung von Handlungsempfehlungen in Bezug auf zukünftige Projekte dieser Art.

\subsection{Wahl eines adäquaten Forschungsdesigns - eine ethnografische Fallstudie}

Aufgrund der Einzigartigkeit eines jeden Projektes und des subjektiv erlebten Prozesses, der dabei beschrieben werden soll, eignen sich für diese Untersuchung qualitative (explorativ-interpretative) Erhebungsmethoden (vgl. Flick et al. 2008: 18ff). Qualitative Forschung zielt darauf ab, dynamische Prozesse zu beobachten, $\mathrm{zu}$ beschreiben und $\mathrm{zu}$ verstehen. Im Gegensatz zur oft als objektiv beschriebenen quantitativen Forschung ist sie eher subjektiv und untersucht Einzelfälle in ihrer natürlich-alltäglichen Situierung. Da es bei dieser Forschungsarbeit um die empirische Beschreibung des Projektprozesses und die Einbeziehung der TN in jenen ging, schien hier ein ethnomethodologischer Forschungsansatz angemessen. Dafür spricht auch die Tatsache, dass es sich um ein Theaterprojekt handelte, bei dem die Projektleiterin gleichzeitig die Forscherin war. Ethnologische Forscher zielen darauf ab, eine kulturelle Gruppe aus der Innenperspektive in ihrer natürlichen Umgebung zu beobachten, 
ohne dabei den Untersuchungsgegenstand zu manipulieren. Dabei wollen sie möglichst viele verschiedene Daten aus unterschiedlichen Perspektiven sammeln, um daraus Hypothesen über ein bestimmtes Phänomen abzuleiten. Dieses Prinzip, das auf den Ethnologen Clifford Geertz zurückgeht, nennt man "Dichte Beschreibung". Geertz zufolge besteht

die Hauptaufgabe der Theoriebildung in der Ethnologie nicht darin [...], abstrakte Regelmäßigkeiten festzuschreiben, sondern darin, dichte Beschreibung zu ermöglichen. Es werden keine allgemeinen Aussagen angestrebt, die sich auf verschiedene Fälle beziehen, sondern nur Generalisierungen im Rahmen eines Einzelfalls. (Geertz 1991:37; vgl. dazu auch Dörnyei 2007:130)

Die Daten, die dabei gewonnen werden, haben unmittelbaren Einfluss auf das Forschungsinteresse und können immer wieder zu neuen Hypothesen und Fragen führen. Ebenso wie der Untersuchungsgegenstand ist der Forschungsprozess dynamisch und in sich zyklisch-reflektiv. Das heißt, dass der Forschende seine Fragen und Hypothesen immer wieder reflektiert und versucht, aus den Daten mit Hilfe hermeneutischer Verfahren Sinn zu konstruieren. Chaudron (1988) zählt die Ethnologie zu den vier grundlegenden Forschungstraditionen innerhalb der Fremdsprachenerwerbsforschung (vgl. in Nunan 1999: 3). Der Fremdsprachenunterricht wird hier als ein hochkomplexer sozialer und kultureller Prozess verstanden, der auf beobachtbaren und nicht-beobachtbaren (mentalen, individuellen) Phänomenen beruht. Das Klassenzimmer ist dabei der kulturelle Kontext (vgl. Dörnyei 2007: 130). Im Hinblick auf die Komplexität dieser Faktoren hat sich daher der Ansatz der Aktions- oder Handlungsforschung etabliert, der Lernprozesse dort untersucht, evaluiert und modifiziert, wo sie stattfinden: im Unterricht selbst.

Aktionsforschung ist die systematische Untersuchung beruflicher Situationen, die von Lehrerinnen und Lehrern selbst durchgeführt wird, in der Absicht, diese zu verbessern." (Altrichter 2007:13)

Ihr Ziel ist es, Lehrern innerhalb der Fremdsprachdidaktik Handlungsempfehlungen an die Hand zu geben, die empirisch begründet sind (vgl. Grotjahn 1993: 237). Demzufolge verbindet der Forschungsprozess Praxis und Theorie sowie Aktion und Reflexion (ebd.: 15) und soll in unserem Fall einen Beitrag zur Evaluation und Weiterentwicklung des projektbezogenen, dramapädagogischen Lernens leisten.

Auch die hier vorgestellte Untersuchung verfolgte das Ziel, mit Hilfe eines offenen Fragebogens, das Projekt im Hinblick auf die Fragestellung durch verschiedene Arten der Datenerhebung sowie aus mehreren Perspektiven zu reflektieren und daraus Handlungsschlüsse zu ziehen. Das Untersuchungsdesign folgte dem Verfahren der empirischen Perspektiventriangulation. Das heißt, dass sowohl TN-Evaluationen (TN-Perspektive) sowie eigene Reflexionen der Forscherin (Eigenperspektive) und Beobachtungen zweier involvierter Expertinnen (Fremdperspektive) in die Erhebung einflossen. Dabei wurden 
Interpretationen zu einem bestimmten Forschungsfokus, die aus einem Datensatz gewonnen wurden, mit anderen Datensätzen konfrontiert. Auf diese Weise konnten konvergente Ergebnisse das Bild des Realitätsausschnittes verdichten, während Divergenzen möglicherweise zu neuen Sichtweisen führten (vgl. Grotjahn 2006: 260).

Bei den Probanden handelte es sich um eine heterogene Deutschlernergruppe aus unterschiedlichen Herkunftsländern mit den Muttersprachen Spanisch (8), Portugiesisch (1), Rumänisch (1) und Englisch (1) sowie unterschiedlichen Deutschkenntnissen und Lernhintergründen. Außerdem nahmen auch vier deutsche Studenten an dem Projekt teil. Die Alterspanne reichte von 19 bis über 29 Jahre. Die erste Expertin war die Co-Leiterin des Projektes Jana Glaser, die das Projekt gemeinsam mit mir initiierte, managte und künstlerisch leitete. Bei der zweiten Expertin handelte es sich um die österreichische Lektorin Romana Radlwimmer, die das Projekt von Anfang an organisatorisch unterstützte, beobachtete und immer mit Rat und Tat zur Seite stand.

Die Konzeption der Fragebögen orientierte sich an Fragekategorien, die sich aus den Vorab-Hypothesen (siehe 4.1) in Folge der Datensichtung und des Reflexionsprozesses ergaben sowie auf der theoretischen Fundierung des Erkenntnisinteresses basierten. Als Frageform wurden offene Fragen gewählt, die auf die subjektive Selbstauskunft der TN abzielten, was die Fähigkeit zur kritischen Selbstreflexion und Selbstevaluation mit einschließt.

\subsection{Datenanalyse und -interpretation}

In der hier vorliegenden Untersuchung wurde ein Methodenmix aus Konzepten zur Quantifizierung von qualitativen Daten und explorativer Inhaltsanalyse vorgenommen. Das heißt, dass die Daten aus den Fragebögen sowohl kategorisiert als auch innerhalb gleicher Antworten ausgezählt und dann wiederum interpretiert wurden. Dabei sollte nicht jeder einzelne Befragte für sich analysiert, sondern die Antworten im Hinblick auf die Fragestellungen und die oben aufgeführten Kategorien als Gesamtergebnis bewertet werden. Die Auswertung war demzufolge eher themengeleitet als personenbezogen. Bei der Kategorisierung und Kodierung der hier vorliegenden Daten wurde ebenfalls ein Methodenmix aus deduktiven und induktiven Verfahren gewählt, wie Altrichter (2007: 195) vorschlägt:

Man sollte alles nutzen, was an begrifflichem Vorverständnis schon vorhanden ist, aber zugleich offen sein für die Überraschungen, die das Datenmaterial bereithält.

Das bedeutet, dass die Antwortkategorien, welche die vorkommenden Ideen klassifizieren, auf einem gewissen Vorverständnis auf der Basis der Theorie und der Fragekategorien beruhten, sich aber gleichzeitig bei der Antwortdurchsicht weiter konkretisierten und veränderten. Nunan schlägt für die Datenauswertung von qualitativen Daten die "Schlüsselwort-Methode“ („key-word-analysis“, 1992: 146) vor, bei der die Antworten der Probanden zunächst in Kategorien 
zusammengefasst und dann nach Häufigkeit der Aussagen ausgewertet werden. Die hier vorliegende Auswertung ging ähnlich vor. Nach dem Sechs-SchrittePrinzip von Altrichter (vgl. 2007: 195f) wurden als erstes in den Antworten alle aussagekräftigen Textstellen im Hinblick auf die Fragestellung markiert und paraphrasiert. Einige TN beantworteten die Fragebögen auf Spanisch. Diese wurden zunächst von der Autorin übersetzt. Die Übersetzung erfolgte dabei so wortgetreu wie möglich, um eine inhaltliche Interpretation zu vermeiden. Die Paraphrasen sind nach den deutschen Übersetzungen entstanden.

Im Anschluss daran wurden diese aussagekräftigen Paraphrasen, die wir hier als ,Ideen' bezeichnen, gesammelt und nach ähnlichen Ideen sortiert, um sie schließlich in Kategorien zu ordnen, die theorie- und fragegeleitet sind. Die Kategorien wurden durch Farben kodiert, sodass die Ideen den Farbkategorien zugeordnet werden konnten. Dabei wurden folgende Antwortkategorien gewählt (siehe Tabelle 1).

Tabelle 1: Antworten nach Faktoren kategorisiert

\begin{tabular}{|c|c|c|c|c|c|}
\hline $\begin{array}{l}\text { Faktor 1: } \\
\text { Deutschler- } \\
\text { nen, } \\
\text { Deutsch- } \\
\text { kontakt, } \\
\text { sprachliches } \\
\text { Handeln }\end{array}$ & $\begin{array}{l}\text { Faktor 2: } \\
\text { Drama- } \\
\text { pädagogik } \\
\text { und } \\
\text { Performative } \\
\text { Kompetenz } \\
\text { (ganzheitli- } \\
\text { ches und } \\
\text { kreatives } \\
\text { Lernen } \\
\text { durch Thea- } \\
\text { terspielen, } \\
\text { Auseinander- } \\
\text { setzen mit } \\
\text { dramati- } \\
\text { scher } \\
\text { Literatur) }\end{array}$ & $\begin{array}{l}\text { Faktor 3: } \\
\text { emotionale } \\
\text { und motiva- } \\
\text { tionale } \\
\text { Aspekte }\end{array}$ & $\begin{array}{l}\text { Faktor 4: } \\
\text { sozio- } \\
\text { kulturelles, } \\
\text { kooperatives } \\
\text { Lernen in } \\
\text { der Gruppe }\end{array}$ & $\begin{array}{l}\text { Faktor 5: } \\
\text { Sonstiges, } \\
\text { Angaben } \\
\text { ohne } \\
\text { Begründung }\end{array}$ & $\begin{array}{l}\text { Faktor 6: } \\
\text { Interesse an } \\
\text { DP aus } \\
\text { beruflichen } \\
\text { Gründen (im } \\
\text { Hinblick auf } \\
\text { DaF-Lehre) }\end{array}$ \\
\hline
\end{tabular}

Praktisch bedeutet dies: Es gab immer eine Einzelidee, die eventuell mehrere Probanden in ähnlicher Weise formuliert hatten. Diese wurde unter einem Überbegriff paraphrasiert und schließlich mit der Farbe der entsprechenden Kategorie versehen, um anschließend alle zu dem Überbegriff passenden Ideen auszuzählen. Auf diese Art und Weise konnten quantitative Aussagen über die Gewichtung einer bestimmten Kategorie getroffen werden. Der Überbegriff musste allerdings nicht zwangsläufig deckungsgleich mit dem Namen der Kategorie sein, sondern vielmehr Elemente dieser im Theorieteil beschriebenen Konstrukte enthalten. Nach diesem Prinzip wurde mit allen Daten verfahren. Tabelle 2 zeigt das Verfahren anhand der ersten Frage: Warum hast du an dem Theaterprojekt teilgenommen? Was war deine Motivation?

Die quantifizierten Daten wurden anschließend in einem dritten Schritt mit 
Tabelle 2: Auswertung der Frage 1 für NMS nach Antwortkategorien

\begin{tabular}{|c|c|c|c|c|c|c|c|}
\hline \multirow{3}{*}{ 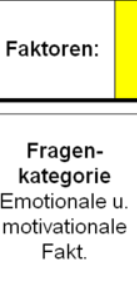 } & $\begin{array}{l}\text { Deutschlernen/ } \\
\text { Deutschkontakt/ } \\
\text { Sprachliches } \\
\text { Handeln } \\
\end{array}$ & $\begin{array}{c}\text { Dramapädagogik } \\
\text { (ganzheitlich, kreativ/ Literatur/ } \\
\text { Performative Kompetenz) }\end{array}$ & $\begin{array}{l}\text { emotionale/ } \\
\text { motivationale } \\
\text { Faktoren }\end{array}$ & \multicolumn{2}{|c|}{$\begin{array}{l}\text { soziokulturelles, } \\
\text { kooperatives Lernen in } \\
\text { der Gruppe }\end{array}$} & \multicolumn{2}{|c|}{$\begin{array}{l}\text { Sonstiges/ } \\
\text { Angaben ohne } \\
\text { Begründung }\end{array}$} \\
\hline & $\begin{array}{l}\text { Ideen, die in Fragen genannt } \\
\text { wurden }\end{array}$ & $\begin{array}{c}\text { Welche } \\
\text { Probanden } \\
\text { haben die Idee } \\
\text { genannt? }\end{array}$ & $\begin{array}{l}\text { Wie v } \\
\text { Probande } \\
\text { die Idee g }\end{array}$ & $\begin{array}{l}\text { ele } \\
\text { haben } \\
\text { nannt? }\end{array}$ & $\begin{array}{r}\text { Zusammenfas } \\
\text { Katego }\end{array}$ & g nach & $\begin{array}{l}\text { Häufigkeit } \\
\text { nach } \\
\text { Kategorien }\end{array}$ \\
\hline & Kontakt mit dt. MS & Proband A,F & 2 & & $\begin{array}{r}\text { Deutschkonta } \\
\text { über }\end{array}$ & Deutsch & 11 \\
\hline \multirow{2}{*}{$\begin{array}{l}\text { Fragengegen- } \\
\text { stand } \\
\text { Motivation zur } \\
\text { Teilnahme am } \\
\text { Projekt }\end{array}$} & Hörverstehen verbessern & Proband A & \multicolumn{2}{|c|}{1} & \multicolumn{2}{|c|}{$\begin{array}{c}\text { Theatererfahrung } \\
\text { sammeln/Freude an } \\
\text { Dramatik }\end{array}$} & 9 \\
\hline & Deutsch anwenden/üben & $\begin{array}{c}\text { Proband } \\
B, C, D, E, F, H, J\end{array}$ & \multicolumn{2}{|c|}{6} & \multicolumn{2}{|c|}{$\begin{array}{l}\text { Neugier/Erfahrungen } \\
\text { sammeln/an positive } \\
\text { Erfahrungen anknüpfen }\end{array}$} & 6 \\
\hline \multirow{8}{*}{$\begin{array}{c}\text { Frage } \\
1\end{array}$} & Theater spielen/lernen/ausprobieren & $\begin{array}{c}\text { Proband } \\
\text { B,C,D,E,F,H,I,J }\end{array}$ & \multicolumn{2}{|c|}{8} & \multicolumn{2}{|c|}{ neue Leute kennenlernen } & 4 \\
\hline & $\begin{array}{l}\text { an positive Theatererfahrungen } \\
\text { anknüpfen }\end{array}$ & Proband E,I,H & \multicolumn{2}{|c|}{3} & \multicolumn{2}{|c|}{ auf Nachfrage } & 2 \\
\hline & Sprachkontakt & Proband B,C & 2 & & & & \\
\hline & $\begin{array}{l}\text { neue Erfahrung sammeln/sich selbst } \\
\text { ausprobieren }\end{array}$ & Proband D,F,K & \multicolumn{2}{|c|}{3} & & & \\
\hline & auf Nachfrage der Projektleiterinnen & Proband D,E & \multicolumn{2}{|c|}{2} & & & \\
\hline & Spanier kennenlernen & Proband $\mathrm{J}$ & \multicolumn{2}{|c|}{1} & & & \\
\hline & Leute kennenlernen & Proband $\mathrm{E}, \mathrm{G}, \mathrm{J}$ & \multicolumn{2}{|c|}{3} & & & \\
\hline & Dt. Dramatiker/Dürrenmatt & Proband $\mathrm{J}$ & \multicolumn{2}{|c|}{1} & & & \\
\hline
\end{tabular}

Hilfe der qualitativen Inhaltsanalyse ausgewertet. Diesen Analysedreischritt beschreibt Mayring (2010: 22) mit „von der Qualität zur Quantität zur Qualität“. Die Daten wurden also am Ende wieder interpretiert, mit dem Ziel, latentes Wissen zu analysieren und neue Hypothesen zu gewinnen.

\section{Zusammenfassung der Ergebnisse}

Das Ergebnis der Auswertung bildete ein aus den Daten generierter Hypothesenkatalog. Die neugenerierten bzw. erweiterten Hypothesen (a-n) wurden in die sie betreffenden Kategorien (siehe 2.3) eingeordnet, wobei eine zusätzliche Kategorie vorangestellt wurde, die sich auf die Projektinitiative und den Projektbedarf bezieht. Eine tabellarische Darstellung des Thesenkatalogs findet sich bei Birnbaum (2011:100ff).

Generierte Hypothesen innerhalb der konstruktgeleiteten Auswertung:

\section{Projektinitiative, Projektbedarf}

a. Das Projekt war bedarfsorientiert und knüpfte an die Interessen der Zielgruppe an, denn es entstand aus einem Mangel an kulturellen Angeboten zum Deutschlernen in der Stadt Salamanca und war gleichzeitig eine kulturelle Reaktion auf die bestehenden gesellschaftlichen Verhältnisse.

Lernerorientierung

Siehe Projektinitiative/Projektbedarf

Lernerautonomie 
Siehe Kategorien 4 und 6

\section{Kooperatives und soziales Lernen}

b. Das kooperative Lernen innerhalb des Projektes förderte die sprachliche Interaktion zwischen den TN. Dadurch konnten die Quantität und Qualität fremdsprachlicher Äußerungen erhöht und Sprechhemmungen abgebaut werden.

Die regelmäßigen Reflexionsrunden sowie die gezielten Theaterspielübungen und gruppendynamischen Spiele verstärkten das Gruppengefühl und halfen den TN, Vertrauen aufzubauen und Sprech- und Spielängste abzubauen.

c. Bei der Durchführung eines fremdsprachlichen Theaterprojektes verändert sich die Rolle des „Lehrers“ hin zu einem Projekt- und Spielleiter, der ein gleichberechtigter Partner und Berater im Lernprozess ist.

d. Bei der Projektarbeit bietet es sich an, die Gruppe im Sinne eines Teamteaching in Zusammenarbeit mit mindestens einem Kollegen zu leiten. Kooperatives Lehren kann ausgeglichenes und demokratisches Leiten fördern, bei dem sich die Lehrenden mit ihrem Wissen, ihren Ideen und ihren Eigenschaften positiv ergänzen können.

e. Die Zusammenarbeit von MS und NMS sowie TN unterschiedlicher Sprachniveaus und Herkunftsländer wird von den TN als bereichernd und lernfördernd empfunden, wenn ein gegenseitiges Voneinander-Lernen stattfindet, das heißt die Rollen zwischen Lernenden und Lehrenden gemäß den Stärken und Schwächen der TN immer wieder wechseln.

\section{Kommunikation und sprachliches Handeln}

f. Die Möglichkeit, innerhalb des Projektprozesses selbst sprachlich zu handeln und Verantwortung zu übernehmen, förderte das autonome Lernen der TN. Autonomes Lernen kann durch gezielte didaktisch/methodische Aufgabenstellungen, die auf aktive sprachliche Partizipation abzielen, gefördert werden.

\section{Ganzheitlichkeit im dramapädagogischen Prozess}

g. DP ist als ganzheitliche Methode besonders geeignet, um fremdsprachliches Lernen zu fördern, da sie handlungsorientiert ist und sich positiv auf affektive und motivationale Faktoren auswirkt.

\section{Reflexion (der Lernprozesse) und Evaluation (des Projekts)}

h. Das Theaterprojekt gab vielen TN das Gefühl, sich sprachlich (z.B. durch Abbau von Sprechängsten) und auch persönlich (z.B. durch einen Perspektivenwechsel) weiterentwickelt zu haben. Die Beschreibung solcher Entwicklungsprozesse erweist sich als problematisch.

i. KL und DP sowie die natürliche Anwendung der Fremdsprache im Handlungskontext in Projekten fördern die sprachliche Interaktion zwischen den TN. Dadurch können die Quantität und Qualität fremdsprachlicher Äußerungen erhöht und Sprechhemmungen abgebaut werden.

j. Die regelmäßige Reflexion und Evaluation des Gruppenprozesses sowie der Einzelleistung in gemeinsamen Gesprächen verstärkten das Gruppengefühl und trieben somit den Projektprozess voran. Dabei ist es wichtig, die TN an diese Methode schrittweise heranzuführen und mit ihnen Zweck und Zielsetzung der 
Methode zu diskutieren.

\section{Emotionale und motivationale Faktoren}

k. Das Theaterprojekt forderte die TN dazu heraus, sich mit persönlichen Ängsten (z.B. Bühnenangst) und Hemmungen (z.B. Sprechhemmungen) auseinanderzusetzen und persönliche Grenzen zu überwinden.

\section{Performative Kompetenz}

1. Die metasprachliche Auseinandersetzung mit dem Dramentext und die eigene kreative Gestaltung des Stückes eröffneten den TN einen tieferen und reflektierten sprachlichen Zugang in den Inszenierungsprozess und eine Auseinandersetzung mit der Inszeniertheit von Wirklichkeit.

\section{Gesellschaftliche Teilhabe und Demokratiegedanke}

m. Die Motivation jedes Einzelnen und der Zusammenhalt der Gruppe wurden positiv durch die gesellschaftliche Öffnung des Projektes über die Theaterproben hinaus, von einem Lern- in einen Lebenskontext, beeinflusst.

\section{Produktorientierung}

n. Das Endprodukt, die gemeinsame Theateraufführung vor Publikum, sowie die individuell und gemeinsam gestalteten Zwischenprodukte wurden von vielen TN als sehr wichtig für die Motivation und das persönliche Engagement eingestuft.

\section{Zusammenfassung:}

Die hier dargelegten Ergebnisse zeigen, dass sich meine Beobachtungen und Vorannahmen (in Form der induktiv gewonnenen Vorab-Hypothesen) in vielen der Aussagen der TN und Expertinnen wiederfanden. Die Daten forderten aber auch die Erweiterung und Modifizierung der Vorab-Hypothesen. Außerdem konnten aus den Daten zahlreiche neue Hypothesen über das kooperative und dramapädagogische Lernen innerhalb des Projektes generiert werden.

Die Antworten machen deutlich, dass es an der USAL einen Bedarf nach und ein Interesse an einem deutschen Theaterprojekt gab und dass sich viele TN auch in Zukunft solche Angebote wünschen. Die Motivation, an dem Projekt teilzunehmen, speiste sich bei den NMS hauptsächlich aus dem Wunsch, sich in ihrer Freizeit mit der deutschen Sprache zu beschäftigen. Weiterhin gab es ein allgemeines Interesse am Theaterspiel als künstlerische Tätigkeit und an einem kulturellen Austausch. Die letzten beiden Faktoren stellen auch für einige MS ein Motiv zur Teilnahme dar. Insgesamt wird das Theaterprojekt von den TN als ein gruppendynamischer und kreativer Prozess des KL beschrieben, in den sich alle Mitglieder individuell und verantwortungsvoll einbrachten und in einer positiven Abhängigkeit das Projektziel verfolgten. So schreibt Proband B (Frage 5):

Am Anfang war ich mir nicht sicher, wie sich die Sache entwickeln würde, ich wusste nicht genau, was da passiert, aber Stück für Stück brachte die Gruppenarbeit und die Kollaboration Ergebnisse. Es war lustig zu sehen, dass sich das Stück nach unseren Ideen und Bedürfnissen entwickelte.

Die regelmäßigen Aktivitäten außerhalb der Proben und die gezielt durchgeführten Theaterspielübungen und gruppendynamischen Spiele halfen gemäß 
den Aussagen dabei, das Gruppengefühl zu stärken und ein Vertrauensverhältnis aufzubauen:

Deshalb war es schön zu bemerken, dass wir jeden Tag unsere Angst und Schüchternheit verloren haben und mehr mitgemacht haben. Die Stimmung war echt super und die Dynamik mittels der Spiele am Anfang jedes Treffens war klasse. (Probandin E, Frage 3)

Ein weiterer wichtiger Punkt, der zur Stärkung der individuellen Leistung sowie der Gruppendynamik beitrug, waren die Reflexionsgespräche innerhalb und außerhalb der Proben sowie die Gruppenkommunikation via E-Mail. Die Antworten einiger TN und der Expertinnen zeigen, dass die gemeinsame Reflexion des Projektprozesses sowie das gegenseitige Feedback und das Lob der Projektleiterinnen und Gruppenmitglieder motivationsfördernd waren und wichtig für die Entwicklung des Projektes sowie für den Abbau von Sprechhemmungen. Bei einigen TN wurde aber auch deutlich, dass sie mit solchen Evaluationsmethoden noch nicht sehr vertraut waren und am Anfang Hemmungen hatten, vor der gesamten Gruppe zu sprechen. Aus diesem Grund bedarf es von Seiten der Projektleiterinnen einer Heranführung und einer Diskussion der Ziele solcher Methoden. Die dramapädagogischen Methoden und die kreative Auseinandersetzung mit der eigenen Rolle werden von den TN ebenfalls als motivationsfördernd beschrieben und haben ihnen Spaß gemacht. Die Funktion der Theaterspielübungen war es vor allem, affektive Ängste wie (Sprach- und Spielhemmungen) abzubauen. Dass dies teilweise gelungen ist, begründen einige Befragte damit, dass immer weniger übersetzt wurde und das Deutschsprechen ganz natürlich geschah, wie es auch die österreichische Lektorin beobachtete:

Bei einzelnen Sprachlernenden, deren Sprachprogression ich längerfristig verfolgt habe, schien mir der Mut zum freien Sprechen deutlich zu wachsen. Meiner Meinung nach lag das daran, dass in Bezug auf Fehler beim mündlichen Ausdruck kein Druck aufgebaut und dass Deutsch als Kommunikationssprache im Projektverlauf zur Normalität wurde.(Radlwimmer, Frage 19).

Viele TN beschreiben auch, wie sie die Fremdsprache zielgerichtet und im Handlungskontext verwendet haben, indem sie selbst zu „Co-Autoren“ (Proband B, Frage 9) des Stückes wurden und sprachlich auf der Bühne und in der Freizeit interagierten.

Um die TN sprachlich aktiv in den Projektprozess einzubeziehen und die Lernerautonomie zu fördern, stellten wir ihnen gezielte sprachliche und kreative Aufgaben, welche die Stück- und Rollenentwicklung vorantreiben sollten. Im Sinne eines handlungsorientierten und zielgerichteten Sprachgebrauchs war das Integrieren von Ideen der TN ein „grundlegendes Prinzip“ (Radlwimmer, Frage 12) des Projektes. Aus diesem Grund verteilten wir Hausaufgaben, bei denen es darum ging, sich zu Hause schriftlich Gedanken zum Stück, aber vor allem zur eigenen Rolle zu machen. Zum Beispiel sollte jeder Akteur eine 
Rollenbiographie zu seiner Studentenrolle entwickeln oder auch seine Kostüme und Requisiten beschreiben. Teilweise sollten sie sich auch kleine Dialoge ausdenken oder ihren Schlussmonolog schreiben. Ausnahmslos alle TN kamen den Aufgaben nach und entwickelten dabei originelle Ideen, die in das Stück einflossen. Die Hausaufgaben wurden per E-Mail an die Projektleiterinnen geschickt, die diese teilweise sprachlich überarbeiteten bzw. kommentierten und zurück schickten. Bei der nächsten Probe wurden die Ideen dann in der Gruppe vorgestellt und besprochen. Durch das Überarbeiten der eigenen Texte und die aktive Spracharbeit wurden die TN sprachlich handelnd tätig.

Alle NMS beschreiben positiven Erfahrungen, die sie beim Deutschlernen durch das Theaterprojekt erfahren haben, sei es in Bezug auf den Abbau von Sprechhemmungen, besseres differenziertes Hören oder einfach die Steigerung der Motivation zum Deutschlernen. Die Antworten zeigen auch, wie wichtig emotionale, motivationale und soziokulturelle Faktoren in Bezug auf das Lernen einer Fremdsprache sind. Die Ergebnisse der NMS machen deutlich, dass es die meisten Deutschlernenden TN gerade am Anfang Überwindung kostete, in der Fremdsprache frei zu sprechen und zu agieren, sie aber mit zunehmender Gruppenbildung und der voranschreitenden Projektentwicklung Sprechhemmungen abbauten. Die Teilnahme von MS am Projekt wird als zusätzlicher motivationaler Faktor beschrieben, da die NMS hier das Gefühl hatten, authentischen Sprachkontakt zu erleben. Proband H schreibt dazu Folgendes (Frage 17):

Auf jeden Fall ist die Anwesenheit von MS bei der Projektdurchführung wesentlich. Die zwei Stunden, die ich in der Woche da war, waren für mich wie eine unmittelbare Reise nach Deutschland jede Woche, weil sie [die MS] unter sich die Sprache mit Natürlichkeit sprachen. Das ist ein Privileg, denn es gibt Leute, die ein Flugzeug nehmen, nur um diese Erfahrung zu haben.

Gleichzeitig zeigen die Antworten der NMS aber, dass bei dieser Konstellation beachtet werden muss, dass das Sprachgefälle von MS zu NMS Sprechhemmungen verursachen kann. Dies hindert ggf. die NMS daran, sich zu äußern, weil sie das Gefühl haben, den MS sprachlich unterlegen zu sein. Dass die MS Deutsch mit Natürlichkeit sprachen, wird zwar als positiv bewertet, führte aber gleichzeitig dazu, dass einige NMS anfangs Probleme hatten, den Gesprächen zu folgen:

Am Anfang sah ich ein sprachliches Hindernis, mit den ganzen MS hatte ich den Eindruck, dass ich eher ein "Laster" sein könnte und obwohl sie mir zeigten, dass das nicht der Fall war, hab ich mich trotzdem manchmal so gefühlt [...] (Proband B, Frage 12).

Aus diesem Grund sollte es Aufgabe der Spiel- und Projektleiterinnen sein, alle TN gemäß ihren Fähigkeiten zu fördern und auf das Sprachniveau zu achten (z.B. indem langsamer gesprochen wird und Zeit zum Nachfragen und Erklären gegeben wird). Dabei sollten die Sprachkenntnisse nicht zum 
Hauptkriterium der gemeinsamen Arbeit gemacht werden. Nicht die Sprache und wer sie dominiert sollte den Projektverlauf gestalten, sondern das gemeinsame Hinarbeiten auf das Handlungsziel, die Inszenierung, die sich neben dem sprachlichen Ausdruck durch vielfältige andere ganzheitliche Ausdrucksmöglichkeiten (Mimik, Gestik, Stimme, Bewegung, Bühnenbild, Kostüme, Requisiten etc.) gestalten lässt. Um es mit den Worten Schewes (1993: 406) zu formulieren:

Die Fremdsprache ist in der Regel nicht der explizite Gegenstand des Unterrichts, vielmehr wird die Fremdsprache verwendet und geübt, während Inszenierungen vorbereitet, durchgeführt und ausgewertet werden. $\mathrm{Da}$ dabei Fehler gemacht werden, wird als natürlicher Vorgang betrachtet. Ja, gerade die sprachliche Risikobereitschaft und die Fähigkeit, mit wenig (sprachlichen) Mitteln viel auszudrücken [...] wird in szenischen Improvisationen bewußt gefördert.

Daher sollte auch die Aufgabenverteilung innerhalb des Gruppenprozesses so sein, dass sich jeder in den Prozess einbringen kann. Auch die Organisation von Requisiten oder die Realisierung einer non-verbalen Tanzszene können wesentlich zum Projekterfolg beitragen. Zusätzliche schriftliche Aufgaben könnten introvertierteren Lernern außerdem helfen, sich sprachlich einzubringen, ohne aus Angst vorm freien Sprechen Ideen zurückzuhalten.

Einige TN beschäftigte auch die Rollenverteilung innerhalb des Stückes. Interessant ist, dass eine NMS betont, dass auch NMS Hauptrollen spielen konnten, während eine andere NMS kritisiert, dass nur die MS die Hauptrollen eingenommen hätten:

Das fand ich echt gut. Um die Sprache zu lernen, finde ich es eigentlich immer besser, mit Muttersprachler zu sprechen. Und obwohl sie natürlich besser sprechen können als wir, die Nichtmuttersprachler, gab es keine Grenze oder Teilung zwischen den Muttersprachler und den Nichtmuttlersprachler. Ich fand es auch echt gut, dass die Muttersprachler die Rolle annehmen könnten, die sehr anspruchsvoll waren." (Proband J, Frage 10, im Original übernommen)

Es gab einen Zusammenhalt, obwohl Deutsche die Hauptrollen spielten und ich glaube es hätte mehr Platz für die ausländischen [TN] geben sollen." (Proband G, Frage 10)

Der Eindruck, dass nur Deutsche die Hauptrollen spielten, erscheint mir hier nicht ganz den Tasachen entsprechend denn es gab auch mehrere NMS, die Hauptrollen bzw. größere Rollen (z.B. die Figuren Newton und Möbius) einnahmen. Zutreffend ist vielmehr, dass diese Teilnehmerin eine "kleinere" Rolle spielte, da sie später zum Projekt hinzustieß und über geringere Sprachkenntnisse verfügte. Die Rollenverteilung erfolgte allerdings in demokratischer Absprache mit den TN. Trotz allem zeigt diese Aussage, wie schwierig es sich für die Projektleiter gestaltet, den Wünschen und Bedürfnissen aller TN gerecht zu werden. 
Die MS hingegen bewerten den kulturellen und sprachlichen Austausch durchweg als positiv und reflektieren auch, dass das Projekt eine authentische Sprachsituation für die NMS darstellte. Probandin L spricht von „einer Art Gruppentandem“, da sowohl Deutsch als auch Spanisch gesprochen wurde. Ein MS schreibt auch, dass er unter anderem zur Gruppenarbeit beigetragen hätte, indem er NMS bei sprachlichen Fragen geholfen hätte. Diese Beispiel beschreibt KL im besten Sinne, da sich die TN untereinander halfen und nicht-deutsche MS von den Kenntnissen der MS profitieren konnten (und auch umgekehrt). Obwohl die Hauptverkehrssprache Deutsch war, gab es auch für die spanischlernenden TN (und auch für die Projektleiterinnen) genügend Möglichkeiten, ihre Sprachkenntnisse anzuwenden und zu vertiefen, wodurch sich auch die Deutschlernenden in der Rolle der Lehrenden wiederfanden. Die Aussagen der TN zur Zusammenarbeit deutscher MS und NMS zeigen allerdings, dass die Rollenverteilung den Sprachkenntnissen zwar angemessen sein muss, aber ebenfalls gerecht und demokratisch erfolgen sollte. NMS dürfen dabei weder sprachlich „vorgeführt“ werden noch sollte man ihnen die Möglichkeit nehmen, über ihre Grenzen hinauszuwachsen. Um ausgeglichenes KL zu gewährleisten, sollte auch hier idealerweise ein stetiger Rollenwechsel zwischen Lernenden und Lehrenden innerhalb der kooperativen Lerngruppe stattfinden, damit sich nicht einige TN den anderen unterlegen fühlen. So hätte bei diesem Projekt noch mehr Spanisch in die Projektarbeit einfließen können, wenn z.B. deutsche MS auf der Bühne Spanisch gesprochen hätten. Auf diese Weise hätte ein noch stärkerer Perspektivenwechsel stattfinden können.

Ein letzter Punkt, der hier in Bezug auf die Teilhabe und das sprachliche Handeln interessiert, ist, wie die TN die E-Mail-Kommunikation mit den Projektleiterinnen einschätzen, da diese der Hauptkommunikationskanal außerhalb der Proben war. Hierüber standen die Projektleiterinnen regelmäßig mit allen TN in Kontakt und organisierten so Probentreffen und andere Termine, dokumentierten die Stückentwicklung und den Probenverlauf, fassten „Erarbeitetes zusammen“ (Glaser, Frage 16), verteilten und kommentierten die Hausaufgaben und motivierten auch durch lobende Worte. So schreibt Glaser (ebd.):

Letztendlich trugen E-Mails an die gesamte Gruppe und die darin konsequent verwendete Anrede („Liebe Physiker") auch positiv zur Bildung des Gemeinschaftsgefühls bei.“

Radlwimmer betont auch die Übungsfunktion der E-Mails in Bezug auf den schriftlichen Ausdruck und die „Kontrolle des eigenen Status quo“ (Frage 16) durch die regelmäßige Rückmeldung und Zusammenfassung des Projektprozesses durch die Spielleiterinnen. Gleichzeitig wäre es aber auch für die TN eine Möglichkeit gewesen, den Projektleiterinnen ein Feedback zu geben (Radlwimmer, ebd.). Die Methode über E-Mails zu kommunizieren, war ein wichtiger didaktischer Schritt, da auf diese Weise nicht nur die Organisation des Projektes erleichtert wurde und so das Projekt auch außerhalb der Proben weiterlief, sondern auch besonders die schriftliche Kommunikation gefördert 
wurde. Außerdem mussten sich die TN aktiv mit ihren Rollenidentitäten auseinandersetzen. Die Ergebnisse zeigen, dass die meisten TN diese Methode positiv bewerten und den Nutzen erkannt haben.

Abschließend soll auf die Worte eines Probanden (M) eingegangen werden, der schreibt: „Der größte Motor des Projekts war die Gruppe selbst“ (Frage 4) und „Eine Theatergruppe steht und fällt mit den Leuten, die daran teilnehmen“ (Frage 7). Natürlich ist den Worten des Probanden nur zuzustimmen, denn jedes Projekt ist hochgradig individuell sowie ein Produkt seiner Mitglieder und sein Erfolg oder Misserfolg wird durch deren Partizipation bestimmt. Trotzdem zeigen die wiederholten Antworten der Probanden, dass sich gewisse didaktische Handlungsschritte auch bei unterschiedlichsten Lernerund Persönlichkeitstypen positiv auf deren eigenes Handeln und damit auch den Lernprozess auswirken können.

\section{Kritische Reflexion der Ergebnisse und Ausblick}

Die Forschungsergebnisse zeigen, dass es sich bei dem Theaterprojekt Entre bastidores mit den Physikern um ein gelungenes Beispiel der Verbindung von KL und DP in Form eines handlungsorientierten Projektes handelte. Die Aussagen der TN und Expertinnen bestätigen, dass bei diesem Projekt eine zielgerichtete, authentische sowie kreative Sprachanwendung des Deutschen als Fremdsprache stattfand. Dabei haben sich die Faktoren des sozialen und kooperativen Lernens zwischen Lernern verschiedener Deutschniveaus sowie MS und NMS innerhalb des kreativen und fiktionalen Kontextes der DP positiv auf motivationale und affektive sowie persönliche Faktoren bei den TN ausgewirkt. Weiterhin demonstrieren die Ergebnisse, dass das Projekt für alle Akteure die Möglichkeit bot, persönliche und sprachliche Grenzen zu überwinden und über den kulturellen und sprachlichen Austausch vielfältige Perspektiven kennenzulernen. Die Studie sollte zeigen, dass diese Faktoren kein bloßes Nebenprodukt einer zufällig harmonierenden Gruppe waren, sondern auf einem didaktisch/methodischen Handlungskonzept fußten, das gezielt auf die Interessen und Bedürfnisse der TN einzugehen versuchte. Dabei wurden den Deutschlernenden fiktionale sowie authentische kommunikative Handlungsräume eröffnet, die sie zum Weiterlernen motivieren sollten. Der Wunsch vieler Probanden nach einem erneuten Projekt dieser Art macht das Potential und die Bedeutung solcher Initiativen für den DaF-Unterricht sowohl in Spanien (vgl. dazu Huth 2009) als auch weltweit deutlich. Die Arbeit soll somit als ein weiterer Vorstoß in eine projektorientierte und dramapädagogische Fremdsprachendidaktik (vgl. Küppers et al. 2011) verstanden werden und will Fremdsprachenlehrende animieren, Initiativen $\mathrm{zu}$ ergreifen und sich den Herausforderungen Projekt und DP zu stellen. Dies bedeutet natürlich, dass im institutionellen Fremdsprachenunterricht Raum und Möglichkeiten für ein solches anwendungsbezogenes Sprachenlernen geschaffen werden müssen. Dies ist nur möglich, wenn von bildungspolitischer Seite anerkannt wird, dass solche Methoden nicht nur eine lernfördernde Wirkung haben 
können, sondern auch wichtig zur Vermittlung von Schlüsselkompetenzen (z.B. „soziale Kompetenz und Bürgerkompetenz“, „Eigeninitiative“ und „kulturelle Ausdrucksfähigkeit"; vgl. Europäische Kommission 2011) sind. Das Lernen soll in diesem Sinne als ein sozialer und lebenslanger Prozess wahrgenommen werden. Aus diesem Grund plädiere ich für eine zunehmende Öffnung des Fremdsprachenunterrichts in einen gesellschaftlichen Kontext, der an die Lebenswelt der Lernenden anknüpft, denn „[...] das Lernen von Sprache braucht das Hinausgreifen in die Welt [...]“ (Bach \& Timm 2009: 37). Dabei können auch die medialen Gewohnheiten der Lernenden (beispielsweise die Lern- und Lebenswelt Internet) für didaktische Zwecke genutzt werden, um sie zur Partizipation zu animieren aber auch um einen kritischen Umgang mit der medialen Wirklichkeit zu thematisieren. Ein weiterer Aspekt, der hier zum Tragen kommen sollte, ist das Prinzip eines kooperativen Lehrens und Leitens fremdsprachlicher Lernformen. Die Arbeit sollte zeigen, dass die Zusammenarbeit mit einem Kollegen nicht nur eine bereichernde Lehrerfahrung sein kann, sondern ebenfalls eine Entlastung bei der Vorbereitung von Unterrichtskonzepten und der Entwicklung von Ideen.

Bei allem Enthusiasmus gegenüber einem Fremdsprachenlernen im Theaterprojekt sollte aber auch deutlich geworden sein, dass sich die empirische Beschreibung von Lernprozessen und didaktischen Arrangements schwierig gestaltet, da es sich um hochgradig komplexe Prozesse handelt, die von vielfältigen Faktoren bestimmt werden. Hinzu kommt die Tatsache, dass es sich bei dem hier vorgestellten Projekt um ein einmaliges Ereignis mit einer individuellen Gruppenzusammensetzung handelte, das sich in dieser Form nicht wiederholen lässt. Aus diesen Gründen konnte die Arbeit den Anforderungen an eine Fremdsprachenerwerbsforschung, die den empirischen Nachweis einer kognitiven Lernprogression fordert, nicht nachkommen. Allein die Behauptung einiger NMS, sie hätten sprachlich etwas gelernt, reicht nicht aus, um einen tatsächlichen Sprachlernzuwachs nachzuweisen. Um eine solche Progression innerhalb einer Aktionsforschung zu hinterfragen, hätte man eine größere Probandengruppe über einen längeren Zeitraum erforschen müssen. Dabei hätte man beispielsweise die Handlungsprodukte der TN sowie deren sprachliche Bearbeitung und Weiterentwicklung untersuchen können bzw. die TN einem sprachlichen Pre- und Posttest unterziehen müssen. Die empirische Untersuchung dramapädagogischer Methoden in Bezug auf kognitive Wirkungsweisen, das heißt, den Zusammenhang zwischen sprachlichem Handeln und dem Erwerb fremdsprachlicher Strukturen, wäre daher sicherlich ein interessantes und ausbaufähiges Forschungsfeld (vgl. dazu auch Even 2003: 295; Bonnet \& Küppers 2011: 43).

Weiterhin wird hier die Problematik deutlich, der ein Lehrender begegnet, wenn er seinen eigenen Unterricht erforschen möchte, da die Doppelrolle eines didaktischen Leiters und Forschers oft nicht einfach zu vereinen ist. Das Hauptziel des Projektes war es zunächst, gemeinsam mit allen TN eine erfolgreiche Theateraufführung zu inszenieren und ein für alle zufriedenstellendes Projekt durchzuführen, das den Bedürfnissen des Einzelnen und der Gruppe gerecht 
wird. Aus diesem Grund stand die Durchführung des Theaterprojektes während dieser Zeit im Vordergrund meiner Tätigkeit als Leiterin, wodurch die Forschungsarbeit beeinträchtigt wurde. Nichtsdestotrotz will sich die Arbeit als Vorstoß in eine handlungsorientierte und praktische Unterrichtsforschung verstanden wissen, der für die Verbindung der didaktischen Konzepte des KL und der DP im Sinne eines handlungsorientierten und kommunikativen Fremdsprachenunterrichts plädiert, um damit den Anforderungen des GeR nachzukommen und

Methoden des modernen Sprachunterrichts zu fördern, die die Unabhängigkeit des Denkens, des Urteilens und des Handelns zusammen mit sozialen Fähigkeiten und Verantwortungsbewusstsein stärken. (Trim 2009: 16)

\section{Literaturverzeichnis}

Altrichter, Herbert; Posch, Peter (2007): Lehrerinnen und Lehrer erforschen ihren Unterricht. Unterrichtsentwicklung und Unterrichtsevaluation durch Aktionsforschung. 4., überarbeitete und erweiterte Auflage. Bad Heilbrunn: Klinkhardt

Bach, Gerhard; Timm, Johannes Peter (2009): Handlungsorientierung als Ziel und Methode. In: Bach, Gerhard \& Timm, Johannes-Peter (Hrsg.): Englischunterricht. Grundlagen und Methoden einer handlungsorientierten Unterrichtspraxis. 1. Auflage. Tübingen: Francke, 1-22

Birnbaum, Theresa (2011): Die Rolle von kooperativem Lernen und Dramapädagogik in Bezug auf das fremdsprachliche Handeln Aktionsforschung zum DaF-Theaterprojekt Entre bastidores mit den Physikern an der Universidad de Salamanca. Masterarbeit in Deutsch als Fremdsprache, Universität Leipzig und Universidad de Salamanca

Bonnet, Andreas; Küppers, Almut (2011): Kooperatives Lernen und Dramapädagogik. In: Küppers, Almut; Schmidt, Torben \& Walter, Maik (Hrsg.): Inszenierungen im Fremdsprachenunterricht. Grundlagen, Formen, Perspektiven. Braunschweig: Julius Klinkhardt, 32-51

Borsch, Frank (2010): Kooperatives Lehren und Lernen im schulischen Unterricht. 1. Auflage. Stuttgart: Kohlhammer

Brandi, Bettina (2006): Grundlagen der Theaterpädagogik. In: Bischoff, Johann \& Brandi, Bettina (Hrsg.): Künstlerisch-technische Grundlagenvermittlung für die Ausbildung im Bereich der angewandten Kultur-, Medien- und Sozialpädagogik. 2., erweiterete Auflage. Aachen: Shaker (Merseburger medienpädagogische Schriften, 1), 76-125

Chaudron, C. (1988): Second language classroom: Research on teaching and learning. New York: Cambridge University Press

Dörnyei, Zoltán (2007): Research methods in applied linguistics. Quantitative, qualitative, and mixed methodologies. Oxford: Oxford University Press 
Even, Susanne (2003): Drama Grammatik. Dramapädagogische Ansätze für den Grammatikunterricht Deutsch als Fremdsprache. München: Iudicium-Verlag

Flick, Uwe; Kardorff, Ernst von \& Steinke, Ines (Hrsg.) (2008): Qualitative Forschung. Ein Handbuch. 6. durchgesehene und aktualisierte Auflage. Reinbek bei Hamburg: Rowohlt-Taschenbuch-Verlag

Flick, Uwe (2007): Qualitative Sozialforschung. Eine Einführung. Vollständig überarbeitete und erweiterte Neuausgabe. Reinbek bei Hamburg: Rowohlt-Taschenbuch-Verlag

Geertz, Clifford (1991): Dichte Beschreibung. Beiträge zum Verstehen kultureller Systeme. Übersetzt von Brigitte Luchesi und Rolf Bindemann. 2. Auflage. Frankfurt am Main: Suhrkamp

Grotjahn, Rüdiger (2006): Zur Methodologie der Fremdsprachenerwerbsforschung. In: Scherfer, Peter \& Wolff, Dieter (Hrsg.): Vom Lehren und Lernen fremder Sprachen. Eine vorläufige Bestandsaufnahme. Frankfurt am Main: Lang, 247-270

Grotjahn, Rüdiger (1993): Qualitative vs. quantitative Fremdsprachenforschung. Eine klärungsbedürftige und unfruchtbare Dichotomie. In: Timm, Johannes-Peter (Hrsg.): Kontroversen in der Fremdsprachenforschung. Dokumentation des 14. Kongresses für Fremdsprachendidaktik. Essen, 7.-9. Oktober 1991, veranstaltet von der Deutschen Gesellschaft für Fremdsprachenforschung (DGFF). Bochum: Brockmeyer, 223-248

Gudjons, Herbert (2008): Handlungsorientiert lehren und lernen. Schüleraktivierung - Selbsttätigkeit - Projektarbeit. 7., aktualisierte Auflage. Bad Heilbrunn: Klinkhardt

Hallet, Wolfgang (2010): Performative Kompetenz und Fremdsprachenunterricht. In: Scenario 1, 5-18

Holl, Edda (2011): SPRACH-FLUSS: Theaterworkshops mit Jugendlichen aus 16 afrikanischen Ländern - Theaterpädagogik zwischen kultureller Bildung und Fremdsprachendidaktik. In: Scenario 2, 13-31

Johnson, David W.; Johnson, Roger T. \& Holubec, Edythe Johnson (1994): Teaching cooperative learning in the classroom. Alexandria, VA: Association for Supervision and Curriculum Development

Koch, Gerd \& Streisand, Marianne (2003): Wörterbuch der Theaterpädagogik. Berlin: Schibri-Verlag

Konrad, Klaus \& Traub, Silke (2005): Kooperatives Lernen. Theorie und Praxis in Schule, Hochschule und Erwachsenenbildung. 2., überarbeitete und ergänzte Auflage. Baltmannsweiler: Schneider-Verl. Hohengehren

Küppers, Almut; Schmidt, Torben \& Walter, Maik (Hrsg.) (2011): Inszenierungen im Fremdsprachenunterricht. Grundlagen, Formen, Perspektiven. Braunschweig: Julius Klinkhardt 
Lutzker, Peter (2007): The Art of Foreign Language Teaching. Improvisation and Drama in Teacher Development and Language Learning. Tübingen: Narr Francke Attempto Verlag GmbH

Mairose-Parovsky, Angela (2000): Interaktionspiele und Transkulturalität. In: Schlemminger, Gerald; Brysch, Thomas \& Schewe, Manfred (Hrsg.):

Pädagogische Konzepte für einen ganzheitlichen DaF-Unterricht. 1. Auflage. Berlin: Cornelsen, 60-71

Meinefeld, Werner (2008): Hypothesen und Vorwissen in der qualitativen Forschung. In: Flick, Uwe; von Kardorff, Ernst \& Steinke,Ines (Hrsg.): Qualitative Forschung. Ein Handbuch. 6. durchgesehene und aktualisierte Auflage. Reinbek bei Hamburg: Rowohlt-Taschenbuch-Verlag, 265-275

Nunan, David (1999): Research methods in language learning. Cambridge: Cambridge University Press

Schewe, Manfred (2007): Drama und Theater in der Fremd- und Zweitsprachenlehre: Ein Blick zurück nach vorn. In: Scenario 1, 131-146

Schewe, Manfred \& Scott, Trina (2003): Literatur verstehen und inszenieren. Foreign Language Literature Through Drama. A Research Project. In: German as a Foreign Language 3, 55-81

Schewe, Manfred (1993): Fremdsprache inszenieren. Zur Fundierung einer dramapädagogischen Lehr- und Lernpraxis. Oldenburg: Zentrum für Pädagogische Berufspraxis Carl-von-Ossietzky-Universität Oldenburg

Schlemminger, Gerald; Brysch, Thomas \& Schewe, Manfred (Hrsg.) (2000): Pädagogische Konzepte für einen ganzheitlichen DaF-Unterricht. 1. Auflage. Berlin: Cornelsen

Schmenk, Barbara (2004): Drama in the Margins? The Common European Framework of Reference and its Implications for Drama Pedagogy in the Foreign Language Classroom. In: German as a Foreign Language 1, 6-23

Slavin, Robert E. (2006): Educational psychology. Theory and practice. 8. Auflage. Boston: Pearson/Allyn \& Bacon

Trim, John L. M.; Quetz, Jürgen; Schieß, Raimund \& Schneider, Günther (2009): Gemeinsamer europäischer Referenzrahmen für Sprachen. Lernen, lehren, beurteilen; [Niveau A1, A2, B1, B2, C1, C2]. [Nachdruck]. Berlin: Langenscheidt

Tschirner, Erwin (2004): Kommunikative Grammatik. - oder wie man lernt, grammatisch richtig zu sprechen -. In: M.J Domínguez, Lübke B. und Mallo A. (Hg.): El alemán en su contexto español. Die deutsche Sprache im spanischen Kontext. Santiago de Compostela, 39-62

Tselikas, Elektra I. (1999): Dramapädagogik im Fremdsprachenunterricht. Zürich: Orell Füssli Verlag AG

Wolff, Dieter (2002): Instruktivismus vs. Konstruktivismus. 20 Thesen zur Lernbarkeit und Lehrbarkeit von Sprachen. In: Bach, Gerhard \& Viebrock, Britta (Hrsg.): Die Aneignung fremder Sprachen. Perspektiven - Konzepte - 
Forschungsprogramm. Frankfurt am Main: Lang (Kolloquium

Fremdsprachenunterricht 10), 19-24

Europäische Kommission (2011): Schlüsselkompetenzen für lebenslanges

Lernen [online verfügbar unter:

http://europa.eu/legislation_summaries/education_training_youth/lifelong_learning/c11090_de.1 zuletzt aktualisiert am 03.03.2011, zuletzt geprüft am 03.07.2012]

Huth, Manfred (2009): Deutsch als Fremdsprache in Spanien.

Bestandsaufnahme und Perspektiven [Online verfügbar unter http:// http://www.manfred-huth.de/fbr/dafdazspa/daf-spa.html, zuletzt aktualisiert am 15.01.2009, zuletzt geprüft am 03.07.2012]

Altrichter, Herbert; Posch, Peter (2007): Lehrerinnen und Lehrer erforschen ihren Unterricht. Unterrichtsentwicklung und Unterrichtsevaluation durch Aktionsforschung. 4., überarbeitete und erweiterte Auflage. Bad Heilbrunn: Klinkhardt

Bach, Gerhard; Timm, Johannes Peter (2009): Handlungsorientierung als Ziel und Methode. In: Bach, Gerhard \& Timm, Johannes-Peter (Hrsg.): Englischunterricht. Grundlagen und Methoden einer handlungsorientierten Unterrichtspraxis. 1. Auflage. Tübingen: Francke, 1-22

Birnbaum, Theresa (2011): Die Rolle von kooperativem Lernen und Dramapädagogik in Bezug auf das fremdsprachliche Handeln Aktionsforschung zum DaF-Theaterprojekt Entre bastidores mit den Physikern an der Universidad de Salamanca. Masterarbeit in Deutsch als Fremdsprache, Universität Leipzig und Universidad de Salamanca

Bonnet, Andreas; Küppers, Almut (2011): Kooperatives Lernen und Dramapädagogik. In: Küppers, Almut; Schmidt, Torben \& Walter, Maik (Hrsg.): Inszenierungen im Fremdsprachenunterricht. Grundlagen, Formen, Perspektiven. Braunschweig: Julius Klinkhardt, 32-51

Borsch, Frank (2010): Kooperatives Lehren und Lernen im schulischen Unterricht. 1. Auflage. Stuttgart: Kohlhammer

Brandi, Bettina (2006): Grundlagen der Theaterpädagogik. In: Bischoff, Johann \& Brandi, Bettina (Hrsg.): Künstlerisch-technische Grundlagenvermittlung für die Ausbildung im Bereich der angewandten Kultur-, Medien- und Sozialpädagogik. 2., erweiterete Auflage. Aachen: Shaker (Merseburger medienpädagogische Schriften, 1), 76-125

Chaudron, C. (1988): Second language classroom: Research on teaching and learning. New York: Cambridge University Press

Dörnyei, Zoltán (2007): Research methods in applied linguistics. Quantitative, qualitative, and mixed methodologies. Oxford: Oxford University Press 
Even, Susanne (2003): Drama Grammatik. Dramapädagogische Ansätze für den Grammatikunterricht Deutsch als Fremdsprache. München: Iudicium-Verlag

Flick, Uwe; Kardorff, Ernst von \& Steinke, Ines (Hrsg.) (2008): Qualitative Forschung. Ein Handbuch. 6. durchgesehene und aktualisierte Auflage. Reinbek bei Hamburg: Rowohlt-Taschenbuch-Verlag

Flick, Uwe (2007): Qualitative Sozialforschung. Eine Einführung. Vollständig überarbeitete und erweiterte Neuausgabe. Reinbek bei Hamburg: Rowohlt-Taschenbuch-Verlag

Geertz, Clifford (1991): Dichte Beschreibung. Beiträge zum Verstehen kultureller Systeme. Übersetzt von Brigitte Luchesi und Rolf Bindemann. 2. Auflage. Frankfurt am Main: Suhrkamp

Grotjahn, Rüdiger (2006): Zur Methodologie der Fremdsprachenerwerbsforschung. In: Scherfer, Peter \& Wolff, Dieter (Hrsg.): Vom Lehren und Lernen fremder Sprachen. Eine vorläufige Bestandsaufnahme. Frankfurt am Main: Lang, 247-270

Grotjahn, Rüdiger (1993): Qualitative vs. quantitative Fremdsprachenforschung. Eine klärungsbedürftige und unfruchtbare Dichotomie. In: Timm, Johannes-Peter (Hrsg.): Kontroversen in der Fremdsprachenforschung. Dokumentation des 14. Kongresses für Fremdsprachendidaktik. Essen, 7.-9. Oktober 1991, veranstaltet von der Deutschen Gesellschaft für Fremdsprachenforschung (DGFF). Bochum: Brockmeyer, 223-248

Gudjons, Herbert (2008): Handlungsorientiert lehren und lernen. Schüleraktivierung - Selbsttätigkeit - Projektarbeit. 7., aktualisierte Auflage. Bad Heilbrunn: Klinkhardt

Hallet, Wolfgang (2010): Performative Kompetenz und Fremdsprachenunterricht. In: Scenario 1, 5-18

Holl, Edda (2011): SPRACH-FLUSS: Theaterworkshops mit Jugendlichen aus 16 afrikanischen Ländern - Theaterpädagogik zwischen kultureller Bildung und Fremdsprachendidaktik. In: Scenario 2, 13-31

Johnson, David W.; Johnson, Roger T. \& Holubec, Edythe Johnson (1994): Teaching cooperative learning in the classroom. Alexandria, VA: Association for Supervision and Curriculum Development

Koch, Gerd \& Streisand, Marianne (2003): Wörterbuch der Theaterpädagogik. Berlin: Schibri-Verlag 
Konrad, Klaus \& Traub, Silke (2005): Kooperatives Lernen. Theorie und Praxis in Schule, Hochschule und Erwachsenenbildung. 2., überarbeitete und ergänzte Auflage. Baltmannsweiler: Schneider-Verl. Hohengehren

Küppers, Almut; Schmidt, Torben \& Walter, Maik (Hrsg.) (2011): Inszenierungen im Fremdsprachenunterricht. Grundlagen, Formen, Perspektiven. Braunschweig: Julius Klinkhardt

Lutzker, Peter (2007): The Art of Foreign Language Teaching. Improvisation and Drama in Teacher Development and Language Learning. Tübingen: Narr Francke Attempto Verlag GmbH

Mairose-Parovsky, Angela (2000): Interaktionspiele und Transkulturalität. In: Schlemminger, Gerald; Brysch, Thomas \& Schewe, Manfred (Hrsg.): Pädagogische Konzepte für einen ganzheitlichen DaF-Unterricht. 1. Auflage. Berlin: Cornelsen, 60-71

Meinefeld, Werner (2008): Hypothesen und Vorwissen in der qualitativen Forschung. In: Flick, Uwe; von Kardorff, Ernst \& Steinke,Ines (Hrsg.): Qualitative Forschung. Ein Handbuch. 6. durchgesehene und aktualisierte Auflage. Reinbek bei Hamburg: Rowohlt-Taschenbuch-Verlag, 265-275

Nunan, David (1999): Research methods in language learning. Cambridge: Cambridge University Press

Schewe, Manfred (2007): Drama und Theater in der Fremd- und Zweitsprachenlehre: Ein Blick zurück nach vorn. In: Scenario 1, 131-146

Schewe, Manfred \& Scott, Trina (2003): Literatur verstehen und inszenieren. Foreign Language Literature Through Drama. A Research Project. In: German as a Foreign Language 3, 55-81

Schewe, Manfred (1993): Fremdsprache inszenieren. Zur Fundierung einer dramapädagogischen Lehr- und Lernpraxis. Oldenburg: Zentrum für Pädagogische Berufspraxis Carl-von-Ossietzky-Universität Oldenburg

Schlemminger, Gerald; Brysch, Thomas \& Schewe, Manfred (Hrsg.) (2000): Pädagogische Konzepte für einen ganzheitlichen DaF-Unterricht. 1. Auflage. Berlin: Cornelsen

Schmenk, Barbara (2004): Drama in the Margins? The Common European Framework of Reference and its Implications for Drama Pedagogy in the Foreign Language Classroom. In: German as a Foreign Language 1, $6-23$ 
Slavin, Robert E. (2006): Educational psychology. Theory and practice. 8. Auflage. Boston: Pearson/Allyn \& Bacon

Trim, John L. M.; Quetz, Jürgen; Schieß, Raimund \& Schneider, Günther (2009): Gemeinsamer europäischer Referenzrahmen für Sprachen. Lernen, lehren, beurteilen; [Niveau A1, A2, B1, B2, C1, C2]. [Nachdruck]. Berlin: Langenscheidt

Tschirner, Erwin (2004): Kommunikative Grammatik. - oder wie man lernt, grammatisch richtig zu sprechen -. In: M.J Domínguez, Lübke B. und Mallo A. (Hg.): El alemán en su contexto español. Die deutsche Sprache im spanischen Kontext. Santiago de Compostela, 39-62

Tselikas, Elektra I. (1999): Dramapädagogik im Fremdsprachenunterricht. Zürich: Orell Füssli Verlag AG

Wolff, Dieter (2002): Instruktivismus vs. Konstruktivismus. 20 Thesen zur Lernbarkeit und Lehrbarkeit von Sprachen. In: Bach, Gerhard \& Viebrock, Britta (Hrsg.): Die Aneignung fremder Sprachen. Perspektiven Konzepte - Forschungsprogramm. Frankfurt am Main: Lang (Kolloquium Fremdsprachenunterricht 10), 19-24

Internetquellen:

Europäische Kommission (2011): Schlüsselkompetenzen für lebenslanges Lernen [online verfügbar unter: http://europa.eu/legislation_summaries/education_training_yout zuletzt aktualisiert am 03.03.2011, zuletzt geprüft am 03.07.2012]

Huth, Manfred (2009): Deutsch als Fremdsprache in Spanien. Bestandsaufnahme und Perspektiven [Online verfügbar unter http:// http://www.manfredhuth.de/fbr/dafdazspa/daf-spa.html, zuletzt aktualisiert am 15.01.2009, zuletzt geprüft am 03.07.2012] 